\title{
Modeling and Forecasting (Un)Reliable Realized Covariances for More Reliable Financial Decisions
}

Tim Bollerslev, Andrew J. Patton and Rogier Quaedvlieg

CREATES Research Paper 2016-10 


\title{
Modeling and Forecasting (Un)Reliable Realized Covariances for More Reliable Financial Decisions
}

This version: April 5, 2016

\author{
Tim Bollerslev ${ }^{\mathrm{a}}$, Andrew J. Patton ${ }^{\mathrm{b}}$, Rogier Quaedvlieg ${ }^{\mathrm{c},}$ \\ ${ }^{a}$ Department of Economics, Duke University, NBER and CREATES \\ ${ }^{b}$ Department of Economics, Duke University \\ ${ }^{c}$ Department of Finance, Maastricht University
}

\begin{abstract}
We propose a new framework for modeling and forecasting common financial risks based on (un)reliable realized covariance measures constructed from high-frequency intraday data. Our new approach explicitly incorporates the effect of measurement errors and time-varying attenuation biases into the covariance forecasts, by allowing the ex-ante predictions to respond more (less) aggressively to changes in the ex-post realized covariance measures when they are more (less) reliable. Applying the new procedures in the construction of minimum variance and minimum tracking error portfolios results in reduced turnover and statistically superior positions compared to existing procedures. Translating these statistical improvements into economic gains, we find that under empirically realistic assumptions a risk-averse investor would be willing to pay up to 170 basis points per year to shift to using the new class of forecasting models.
\end{abstract}

Keywords: Common risks; realized covariances; forecasting; asset allocation; portfolio construction.

JEL: C32, C58, G11, G32

\footnotetext{
${ }^{3}$ The research was partially supported by a grant from Inquire Europe. Bollerslev also gratefully acknowledges the support from CREATES funded by the Danish National Research Foundation (DNRF78). Quaedvlieg was financially supported by the Dutch Organization for Scientific Research (NWO). We would also like to thank Sébastien Laurent, Peter Schotman, along with seminar participants at Erasmus University, Aix-Marseille University, Warwick Business School and Nova SBE Lisbon for helpful comments. Bingzhi Zhao kindly provided us with the cleaned high-frequency data underlying our empirical investigations.
} 


\section{Introduction}

The presence of common risk factors plays a crucial role in the theory and practice of finance. Common risks, and predictions thereof, are typically quantified through return covariances. While inherently unobservable, there is extensive empirical evidence to support the idea that covariances of asset returns vary through time. From a practical estimation and forecasting perspective, this naturally poses a trade-off between the use of long historical samples to accurately estimate the latent covariances, potentially biasing the forecasts if the risks are changing, or restricting the estimation to more recent observations to better capture the short-term dynamics, thereby potentially increasing the estimation and forecast errors. In response to this, we develop a new framework for more accurately forecasting dynamically varying covariances by explicitly incorporating the effects of (un)reliable past covariance measures into the forecasts. Applying the new procedures in asset allocation decisions, we show that these statistical improvements in the accuracy of the covariance forecasts translate into sizeable economic gains for a representative risk averse investor seeking to minimize the overall risk or the tracking error of her portfolio.

Modeling and forecasting of dynamically varying covariances have received much attention in the literature, with numerous multivariate $\mathrm{ARCH}, \mathrm{GARCH}$ and stochastic volatility specifications been proposed for the job. All of these procedures effectively treat the covariances as latent. More recently, however, the increased availability of reliable high-frequency intraday asset prices have has spurred somewhat of a paradigm shift based on the idea of directly modeling and forecasting expost realized covariance measures constructed from intraday data (see, e.g., Andersen, Bollerslev, Christoffersen, and Diebold, 2013, for a discussion of both the earlier parametric models and the more recent realized volatility literature). The benefits of high-frequency-based realized volatility procedures for practical investment and portfolio allocation decisions have also been extensively documented in the literature (e.g., Fleming, Kirby, and Ostdiek, 2003; Bandi, Russell, and Zhu, 2008; Pooter, Martens, and Dijk, 2008; Liu, 2009; Varneskov and Voev, 2013; Hautsch, Kyj, and Malec, 2015, among others).

Even though the use of high-frequency intraday data generally allows for the construction of more accurate realized covariance measures than lower frequency (e.g., daily) data, they are still estimates and as such subject to estimation error. Correspondingly, the use of these covariance estimates in dynamic forecasting models leads to a classical errors-in-variables problem and an attenuation of the parameters towards zero relative to a forecasting model based on the true (latent) covariances. If the measurement errors were homoskedastic, this attenuation would be time invariant, and from a practical forecasting perspective inconsequential. If, however, the measurement errors are heteroskedastic, as we show is the case with realized covariance estimates, all observations are "not equal," and the presence of such heteroskedastic measurement errors can indeed have consequences for forecasting. A covariance estimate with below average measurement error provides a stronger signal and should be more informative about future covariances than an estimate with an 
above average measurement error.

Extending the basic idea and simple univariate volatility models in Bollerslev, Patton, and Quaedvlieg (2016), we propose a new class of multivariate realized covariance based forecasting models that explicitly take into account the influence of measurement errors. To do so, we rely on the asymptotic distribution theory for high-frequency realized covariance estimation (BarndorffNielsen and Shephard, 2004; Barndorff-Nielsen, Hansen, Lunde, and Shephard, 2011) to help guide the magnitude of the time-varying attenuation in the parameters of the models: the parameters should be relatively large on days when the realized covariances are precisely estimated and more heavily attenuated on days when the measurement errors are large and the signals are weak. By contrast, the average attenuation bias implicit in conventional constant parameter models will be too large (small) on days when the realized covariances are (im)precisely estimated.

Our empirical investigations are based on high-frequency data for a sample of ten individual stocks together with a variety of evaluation criteria and out-of-sample methods. Based on standard statistical tests and model confidence sets, we firstly show that the new models systematically beat their constant attenuation benchmarks in the sense of providing covariance matrix forecasts that are significantly closer to the ex-post covariances. ${ }^{1}$

Next, in an effort to underscore the practical relevance of the forecast improvements afforded by the new class of models, we evaluate their performance in portfolio allocation decisions. The detrimental impact of measurement errors in the context portfolio construction has already been widely studied in the literature, and it is well established that the use of ill conditioned and/or poorly estimated covariance matrices often lead to extreme positions far away from the ex-post optimal portfolio weights (see, e.g., Li, 2015, and the many references therein). These extreme positions also typically result in excessively high turnover and transaction costs. One popular strategy to help mitigate these problems, and prevent the portfolio optimizers from "reading too much into the data," is to directly constrain the portfolio weights. Jagannathan and Ma (2003), for instance, advocate the use of no short-sale constraints, while DeMiguel, Garlappi, Nogales, and Uppal (2009a) and Brodie, Daubechies, De Mol, Giannone, and Loris (2009) propose L-norm constrained portfolios. Another popular strategy is to "shrink" the covariance matrix estimates to indirectly help control the portfolio weights. Ledoit and Wolf (2003, 2004a,b), in particular, recommend shrinking the unconditional sample covariance matrix estimate towards some "target" matrix based on a simple factor structure or some other pre-determined matrix. ${ }^{2}$

We propose a new approach that can be interpreted as a dynamic shrinkage procedure. Instead of exogenously shrinking the unconditional covariance matrix estimate, we provide a dynamic alternative in which the covariance matrix forecasts are endogenously shrunk away from the conditional

\footnotetext{
${ }^{1}$ We further document that these improvements in forecast accuracy are not restricted to the attenuation of especially noisy covariance estimates, but occur in response to both reliable and unreliable covariances.

${ }^{2}$ Numerous other combination and related Bayesian model averaging and learning approaches have also been proposed in the literature to help improve on the standard portfolio allocation procedures; see, e.g., Tu and Zhou (2011) and Anderson and Cheng (2016) and the many additional references therein.
} 
to the unconditional covariance matrix with a time-varying shrinkage intensity that depends on the degree of (time-varying) measurement error. Consistent with the idea that the use of shrinkage techniques generally results in less extreme and more stable portfolio allocations, applying the new procedures in the construction of minimum variance portfolios using simulated data leads to positions that are systematically closer to the optimal positions than those implied by otherwise identical benchmark models without any dynamic shrinkage. Importantly, turnover is also reduced substantially compared to the positions for the benchmark models.

Our empirical analysis involves returns on ten stocks from the Dow Jones Industrial Average. Relying on the utility-based framework of Fleming, Kirby, and Ostdiek (2001, 2003), we find that in the absence of any transaction costs, a risk averse investor seeking to minimize the daily variance of her portfolio would be willing to sacrifice up to 140 basis points annually to switch to the more accurate forecasts from our dynamic attenuation model compared to the forecasts obtained from the same model without any attenuation effects. Incorporating empirically realistic transaction costs results in additional gains of 20-70 basis points per year stemming from the reduced turnover. These same qualitative gains hold true for an investor seeking to minimize the tracking error of her portfolio relative to an $\mathrm{S} \& \mathrm{P} 500$ benchmark portfolio. These utility gains remain intact after imposing no short-sales constraints. They also remain over longer weekly and monthly investment horizons, although the relative magnitudes of the gains tend to decrease with the horizon.

The utility benefits obtained from implementing the new models at the daily horizon also exceed the benefits obtained from implementing the models at the weekly and monthly frequencies, despite the increased turnover. In contrast, but consistent with past research, the utility from benchmark models that do not adjust for the time-varying estimation errors in the realized covariances are not generally higher at the daily level, underscoring the detrimental impact of unbalanced positions and "too much trading" stemming from the use of more conventional covariance forecasting procedures. Moreover, we show that our proposed new models compare favorably with existing portfolio allocation procedures, e.g. that of Ledoit and Wolf (2003), intended to counter the adverse effects of estimation errors discussed above.

The remainder of the paper is organized as follows. Section 2 sets up the notation and motivation for the new class of models. Section 3 defines the specific parametric models that we rely on our empirical applications. Section 4 discusses the data. Section 5 presents our estimates of the different models, along with various statistical in- and out-of-sample forecast comparisons. Section 6 discusses the metrics and utility-based approach that we rely on in our economic assessments of the new procedures. Our main empirical findings are presented in Section 7. Section 8 extends our main results to other forecast horizons and also compare the new dynamic attenuation models to other already existing static shrinkage procedures. Section 9 concludes. Additional technical details and empirical results are deferred to four appendices. 


\section{Motivation and realized covariance estimation}

Estimated realized covariances can be decomposed into the sum of two components: the latent true covariance and a measurement error term. Since the latter represents "noise," the time series of realized covariances will appear less persistent than they truly are. As a result the estimated parameters in conventional autoregressive models for the realized covariances are attenuated towards zero. When the measurement error is homoskedastic, the degree of attenuation is proportional to the measurement error variance, but when the measurement error is heteroskedastic, the parameter estimates will be attenuated based on the average magnitude of the measurement errors. Consequently, the attenuation is never optimal: it is too strong (weak) when the past realized covariances are (im)precisely estimated.

In most errors-in-variables settings, the distributions of the measurement errors are unknown. In the context of realized covariance estimation, however, the covariances of the measurement errors may be estimated on a day-to-day basis using theoretical developments in Barndorff-Nielsen and Shephard (2004) and Barndorff-Nielsen, Hansen, Lunde, and Shephard (2011). By directly incorporating an estimate of the magnitude of the errors into the formulation of realized covariance based forecasting models, it is possible to dynamically attenuate the parameters of the models, and in turn improve on the forecasts.

The discussion below briefly summarizes the basic theoretical arguments underlying this dynamic attenuation approach. We purposely keep the setup below as simple as possible to convey the main ideas and intuition.

\subsection{Realized covariances}

To set out the notation, consider the $N$-dimensional log-price process,

$$
P(s)=\int_{0}^{s} \mu(u) d u+\int_{0}^{s} \sigma(u) d W(u)
$$

where $\mu(u)$ and $\sigma(u)$ denote the instantaneous drift and volatility processes, respectively, with corresponding spot covariance matrix $\Sigma(u) \equiv \sigma(u) \sigma(u)^{\prime}, W(u)$ is an $N$-dimensional vector of independent Brownian motions, and the unit time interval is normalized to a day. We exclude jumps for simplicity, but the same ideas readily extend to price process that contain jumps. Following advances in the realized volatility literature, we are interested in modeling and forecasting the daily ex-post covariation, or the daily integrated covariance (see, e.g., the discussion in Andersen, Bollerslev, Diebold, and Labys, 2003). For the price process in equation (1) the integrated covariance for day $t$ simply equals,

$$
\Sigma_{t}=\int_{t-1}^{t} \Sigma(u) d u
$$

The integrated covariance is not directly observable. However, it may be consistently estimated based on ever-finer sampled high-frequency intraday data. 
In addition to the practical market microstructure complications that plague the estimation of integrated variances (see, e.g., the discussion in, Hansen and Lunde, 2006) the practical estimation of integrated covariances is further complicated by the Epps (1979) effect and non-synchronous price observations. A number of alternative estimators have been proposed in the literature to circumvent these complications. In our empirical analysis below, we rely on the Multivariate Kernel (MK) of Barndorff-Nielsen, Hansen, Lunde, and Shephard (2011), for which the asymptotic variance of the measurement errors can easily be estimated; further details about the MK estimator and its asymptotic distribution are provide in Appendix A below. However, the same basic ideas carry over to any consistent estimator for $\Sigma_{t}$, say $S_{t}{ }^{3}$

To facilitate the discussion, let $\varsigma_{t} \equiv$ vech $\Sigma_{t}$ denote the $N^{*}=N(N+1) / 2$ dimensional vectorized version of the true latent integrated covariance matrix of interest $\Sigma_{t}$. Similarly, let $s_{t} \equiv$ vech $S_{t}$ denote the vectorized version of the $S_{t}$ realized covariance matrix estimator. We will refer to the $N^{*} \times N^{*}$ covariance matrix for the corresponding measurement error vector $\eta_{t}=s_{t}-\varsigma_{t}$ by $\Pi_{t}$. The exact form of $\Pi_{t}$ obviously depends on the specific estimator $S_{t}$. For the MK estimator that we rely on in the empirical analysis below, $\Pi_{t}$ is proportional to the so-called integrated quarticity,

$$
\Pi_{t} \propto \int_{t-1}^{t} \Sigma(u) \otimes \Sigma(u) d u
$$

where $\otimes$ denotes the Kronecker product. ${ }^{4}$ In parallel to the integrated covariance $\Sigma_{t}$, the integrated quarticity matrix may be consistently estimated using high-frequency intraday data. In the empirical results reported below, we rely on the specific estimator proposed by Barndorff-Nielsen, Hansen, Lunde, and Shephard (2011). In the following we will refer to the resulting (up-to-scale) measurement error covariance matrix estimates as $\Pi_{t}$ for short.

\section{2. (Un)reliable covariances and attenuation biases}

The effect of measurement errors in univariate autoregressive models has been extensively studied in the literature (see, e.g., Staudenmayer and Buonaccorsi, 2005). In the multivariate setting the problem is less well-developed and the literature have mostly focussed on the effect of measurement errors for identification and hypothesis testing (see, e.g., Holtz-Eakin, Newey, and Rosen, 1988; Komunjer and Ng, 2014). Even so, it is well-established that the autoregressive parameter estimates are attenuated towards zero, both in the uni- and multivariate setting.

In order to provide some intuition for this result and our new dynamic attenuation approach, assume for simplicity that the true (latent) vectorized integrated covariance $\varsigma_{t}$ follows a VAR(1)

\footnotetext{
${ }^{3}$ Other "noise" robust consistent covariance estimators include Aït-Sahalia, Fan, and Xiu (2010), and Christensen, Kinnebrock, and Podolskij (2010).

${ }^{4} \mathrm{~A}$ similar result holds true for other realized covariance matrix estimators. Instead of relying on explicit analytical asymptotic expressions for estimating $\Pi_{t}$, following Gonçalves and Meddahi (2009) the variance of the measurement errors may alternatively be estimated by bootstrap procedures.
} 
model,

$$
\varsigma_{t}=\Phi_{0}+\Phi_{1 \varsigma_{t-1}}+u_{t},
$$

where $\Phi_{0}$ is of dimension $N^{*}$, and the autoregressive parameter $\Phi_{1}$ is of dimension $N^{*} \times N^{*}$. Even though this does not necessarily correspond to the exact discretization of the underlying continuoustime model in equation (1), a simple first-order VAR may still provide a good approximation in many situations (see also the related discussion in Andersen, Bollerslev, and Meddahi, 2004).

In practice, of course, $\varsigma_{t}$ is not observable, so the researcher must estimate any given model using realized covariances, $s_{t}$. Suppose that the researcher relies on the identical $\operatorname{VAR}(1)$ formulation, ${ }^{5}$

$$
s_{t}=\Theta_{0}+\Theta_{1} s_{t-1}+\epsilon_{t} .
$$

For simplicity, assume that the "structural" errors $u_{t}$ and the measurement errors $\eta_{t}=s_{t}-\varsigma_{t}$ are both i.i.d. and uncorrelated. (This, of course, would not be the case for the realized covariances if the underlying $\sigma(u)$ process is time- varying.) Under these simplifying assumptions, it is straightforward to show that the OLS estimate of $\Theta_{1}$ will be functionally related to the population value of $\Phi_{1}$ by,

$$
\begin{aligned}
\widehat{\Theta}_{1} & =\left(\mathbf{s}^{\prime} \mathbf{s}\right)^{-1}\left(\boldsymbol{\varsigma}^{\prime} \boldsymbol{\varsigma}\right) \Phi_{1} \\
& =\left(\boldsymbol{\varsigma}^{\prime} \boldsymbol{\varsigma}+\boldsymbol{\eta}^{\prime} \boldsymbol{\eta}\right)^{-1}\left(\boldsymbol{\varsigma}^{\prime} \boldsymbol{\varsigma}\right) \Phi_{1},
\end{aligned}
$$

where $\mathbf{s}=\left(s_{1}, \ldots, s_{T-1}\right)$ denotes the $T-1 \times N^{*}$ matrix of lagged realized covariances, with $\varsigma$ and $\boldsymbol{\eta}$ defined analogously. The actual estimated parameter matrix therefore equals the population parameter matrix times the ratio of the variation of the true latent process divided by the variation of the estimated process. ${ }^{6}$ This latter ratio is commonly referred to as the reliability ratio. As this relationship makes clear, depending on the covariance structure of $\eta$, certain parameters may be biased towards zero, while other parameters may be biased away from zero. However, regardless of the direction, the bias is always proportional to the magnitude of the measurement errors.

The bias implied by equation (5), could be removed based on an estimate for the reliability ratio to arrive at an unconditionally unbiased estimate for the "structural" $\Phi_{1}$ parameters. Although these parameters may sometimes be of interest in their own right, from a practical forecasting perspective it is the $\Theta_{1}$ parameters and the dynamic dependencies in the actually observed realized covariances $s_{t}$ that matter. It follows from the expression in equation (5) that even if the $\Phi_{1}$ parameters are time-invariant, as long as the measurement errors are heteroskedastic, the "optimal" $\Theta_{1}$ parameters to be used for forecasting purposes should be dynamically attenuated to reflect the

\footnotetext{
${ }^{5}$ In the univariate case the population parameters of an $\operatorname{AR}(p)$ model with homoskedastic measurement errors can easily be identified by estimating an $\operatorname{ARMA}(p, p)$ model. In the multivariate case the composite error term $\epsilon_{t}-\eta_{t}+\Theta_{1} \eta_{t-1}$ is typically not a finite order moving average process, so a finite dimensional VARMA model will not identify the population parameters.

${ }^{6}$ In the univariate case the relationship simplifies to the easier to interpret $\widehat{\Theta}_{1}=\left(1+\operatorname{Var}\left(\eta_{t}\right) / \operatorname{Var}\left(\varsigma_{t}\right)\right)^{-1} \Phi_{1}$, where the estimated parameter equals the population parameter times a noise-to-signal ratio.
} 
temporal variation in the reliability of the past realized covariance measures.

The specific expression in equation (5) is based on a number of simplifying assumptions and merely meant to illustrate the main idea. A number of important choices need to be made in the way in which the dynamic attenuation idea is actually implemented in practice. Firstly, a $\operatorname{VAR}(1)$ model is likely not the best specification for characterizing the dynamic dependencies in $s_{t}$. Secondly, reliable estimation of all the elements in the $\Pi_{t}$ matrix on a period-by-period basis, not to mention the inverse, presents some formidable challenges, even for moderately large $N$. The next section discusses the dynamic attenuation models that we rely on in our actual empirical investigations.

\section{Dynamic attenuation models}

The specification of empirically realistic, yet practically feasible, multivariate realized volatility models raises a number of important practical issues. Motivated by the discussion in the previous section, we propose new parsimonious dynamic specifications for the high-frequency realized covariances, in which we allow the autoregressive parameters of the models to depend linearly on the measurement errors of covariance matrix estimates. We purposely restrict the parameterizations to relatively simple scalar formulations, while allowing each element of the covariance matrix to exhibit its own distinct dynamic dependencies as a function of the measurement errors variances.

We consider three popular baseline models: the vech-HAR (Chiriac and Voev, 2010), the HARDRD model (Oh and Patton, 2015), and the HEAVY model (Noureldin, Shephard, and Sheppard, 2012). For comparison purposes, we also consider a simple Exponentially Weighted Moving Average (EWMA) filter, in which we allow the filter weights to vary with the estimation error. It is not our goal to run a horse-race between the various models. Instead, we seek to illustrate how the basic approach may be implemented quite generally, and in turn evaluate the performance of the models with dynamically attenuated parameters relative to the otherwise identical models with constant parameters.

\subsection{HARQ models}

The Heterogeneous AutoRegressive (HAR) model of Corsi (2009) has arguably emerged as the most widely used realized volatility-based forecasting model. The model was first extended to a multivariate setting by Chiriac and Voev (2010). The scalar version of the vech HAR model is formally defined by

$$
s_{t}=\theta_{0}+\theta_{1} s_{t-1}+\theta_{2} s_{t-5 \mid t-1}+\theta_{3} s_{t-22 \mid t-1}+\epsilon_{t},
$$

where $s_{t-h \mid t-1}=\frac{1}{h} \sum_{i=1}^{h} s_{t-i}$ denote the vectorized version the $h$-day realized covariance matrix. The intercept $\theta_{0}$ is a $N^{*} \times 1$ dimensional vector, while the $\theta_{1}, \theta_{2}$ and $\theta_{3}$ parameters are all assumed to be scalar. This simple specification is highly parsimonious and readily ensures that the covariance matrix forecasts are positive definite. 
The standard vech HAR formulation in (6) does not take into account the fact that the lagged realized covariances are measured with error. Bollerslev, Patton, and Quaedvlieg (2016) proposed a simple modification to accommodate this in the context of univariate volatility models, by allowing $\theta_{1}$ in (6) to depend on an estimate for the measurement error variance: $\theta_{1, t}=\left(\theta_{1}+\theta_{1 Q} R Q_{t}^{1 / 2}\right)$, where $R Q_{t}$ denotes the daily realized quarticity. Following the discussion in the previous section, the resulting "HARQ" model may be interpreted as a linear approximation to the inverse of (5) in the univariate setting. ${ }^{7}$ This same idea may be extended to multivariate settings. In such settings, measurement errors may be of even greater importance, given the growth in the number of estimated quantities, however in these cases it is also more difficult to accurately estimate the magnitude of the measurement errors. Correspondingly, it is imperative to strike a balance between the difficulties in accurately estimating all of the elements in $\Pi_{t}$ and the way in which the dynamic attenuation of the autoregressive parameters is actually implemented.

In particular, as for the covariance matrix $s_{t}$ itself, it is generally easier to accurately estimate the diagonal elements of $\Pi_{t}$ than the off-diagonal covariance elements. Hence, we focus on $\pi_{t} \equiv$ $\sqrt{\operatorname{diag}\left(\Pi_{t}\right)}$, the vector of asymptotic standard deviations for each of the individual element in the $S_{t}$ covariance matrix. The correspondingly modified vech HARQ model estimated below takes the form, ${ }^{8}$

$$
\begin{aligned}
s_{t} & =\theta_{0}+\theta_{1, t} \circ s_{t-1}+\theta_{2} s_{t-5 \mid t-1}+\theta_{3} s_{t-22 \mid t-1}+\epsilon_{t}, \\
\theta_{1, t} & =\theta_{1} \iota+\theta_{1 Q} \pi_{t-1},
\end{aligned}
$$

where $\circ$ denotes the Hadamard product, $\iota$ is an $N^{*}$ dimensional vector of ones, and $\theta_{1}$ and $\theta_{1 Q}$ are scalar parameters. More general specifications could, of course, be implemented, but the parsimonious formulation in (7) is shown in our out-of-sample analyses to perform well. Also, note that even though the $\theta_{1}$ and $\theta_{1 Q}$ parameters are both assumed to be scalar, the resulting $\theta_{1, t}$ parameter vector allows for different dynamics in each of the individual elements in the covariance matrix based on their own measurement error variances. ${ }^{9}$ Given the intuition underlying this model, we expect $\theta_{1}$ to be larger than would be found in a conventional HAR model, and we expect $\theta_{1 Q}$ to be negative, so that realized covariance matrices that are measured with greater error are given lower weight in the forecasting model.

\subsection{HARQ-DRD models}

An alternative approach to possibly allow for more general dynamic dependencies, while ensuring positive definite covariance matrix forecasts, is to model the variances and correlations

\footnotetext{
${ }^{7}$ The $\mathrm{Q}$ suffix refers to the use of the quarticity measure to guide the attenuation.

${ }^{8}$ In all of the empirical results reported below, we demean the $\pi_{t}$ vector to render the $\theta_{1}$ coefficients directly interpretable as the value at the average measurement error level.

${ }^{9}$ The $\theta_{2}$ and $\theta_{3}$ parameters could similarly be allow to depend on the measurement errors in $s_{t-5 \mid t-1}$ and $s_{t-22 \mid t-1}$, respectively. However, the magnitude of the errors generally decrease with the horizon, and the difficulties in accurately estimating the integrated quarticity may easily outweigh the benefits of adjusting the weekly and monthly coefficients; Bollerslev, Patton, and Quaedvlieg (2016) provides an analysis of the corresponding tradeoffs in the univariate context.
} 
separately. The HAR-DRD model of Oh and Patton (2015), is based on the decomposition of a covariance matrix into

$$
S_{t}=D_{t} R_{t} D_{t}
$$

where $D_{t}$ denotes the diagonal matrix of standard deviations, and $R_{t}$ is the correlation matrix, see Bollerslev (1990). In the HAR-DRD model, the individual variances are modeled by univariate HAR models, and the conditional correlation matrix is modeled using a scalar multivariate HAR model. The HAR-DRD model is readily extended to allow for dynamic attenuation effects by incorporating the influence of measurement errors into the parameters in the models for the variances and/or correlations.

In the HARQ-DRD model analyzed below, we rely on the univariate HARQ model of Bollerslev, Patton, and Quaedvlieg (2016), defined analogously to the multivariate model in equation (7) above, together with the scalar multivariate HAR in equation (6) for modeling the vectorized correlation matrix. It would of course be possible to also consider a HARQ specification for the correlations. However, the heteroskedasticity in the measurement errors for the correlations tend to be somewhat limited, likely outweighing the benefits of attenuating the autoregressive correlation parameters. ${ }^{10}$

The parameters in the vech $\operatorname{HAR}(\mathrm{Q})$ and the $\operatorname{HAR}(\mathrm{Q})$-DRD models discussed above are easily estimated by standard OLS procedures. The estimation of the parameters in the HEAVY(Q) models, which we discuss next, require the use of more complicated (pseudo-)maximum likelihood techniques and non-linear optimization procedures.

\subsection{HEAVYQ models}

The multivariate HEAVY class of models was originally introduced by Noureldin, Shephard, and Sheppard (2012). In contrast to the HAR models discussed above, which are only meant to forecast the daily covariances, HEAVY models are designed to characterize the entire conditional daily return distribution, explicitly incorporating information in past realized covariances.

To set out the basic idea, let $V_{t}=E\left(r_{t} r_{t}^{\prime} \mid \mathcal{F}_{t-1}\right)$ denote the conditional covariance matrix for the daily returns $r_{t}$, where the time $t-1$ information set $\mathcal{F}_{t-1}$ includes all of the past realized covariances. Correspondingly, let $v_{t}=$ vech $V_{t}$ denote the vectorized version of the daily conditional covariance matrix. The HEAVY model with covariance targeting that we rely on for modeling $v_{t}$ below, may then be expressed as,

$$
v_{t}=\left(I_{d^{*}}-b-a \kappa\right) \lambda_{V}+b v_{t-1}+a s_{t-1},
$$

where $a$ and $b$ are scalar parameters, and $\kappa$ serves to adjust the expectation of the high-frequency intraday covariance matrix to match the unconditional expectation of the daily covariance matrix

\footnotetext{
${ }^{10}$ Following Barndorff-Nielsen and Shephard (2004) and assuming a constant spot volatility for simplicity, it is possible to show that the asymptotic standard deviation for the correlation coefficient $\rho_{t}$ approximately equals $1-\rho_{t}^{2}$, which invariably is limited by $\left|\rho_{t}\right| \leq 1$.
} 
$\lambda_{V} .{ }^{11}$ The model's parameters can be estimated using standard quasi-maximum likelihood techniques. To facilitate the practical implementation, we rely on the composite likelihood approach of Pakel, Shephard, Sheppard, and Engle (2014), which is easy to implement in large dimensions; further details are provided in Appendix C.

The conventional HEAVY model in (9) does not account for estimation errors in the realized covariance estimates. Following the same approach used in the specification of the vech HARQ and HARQ-DRD models above, the HEAVY model may similarly be adapted to allow the impact of $s_{t-1}$ to vary over time depending on the degree of measurement error,

$$
\begin{aligned}
v_{t} & =\left(I_{d^{*}}-b-a_{t} \kappa\right) \lambda_{V}+b v_{t-1}+a_{t} \circ s_{t-1}, \\
a_{t} & =a \iota+a_{Q} \pi_{t-1},
\end{aligned}
$$

where again $a_{Q}$ is assumed to be scalar. We will refer to this specification as the HEAVYQ model below.

\subsection{EWMAQ filters}

Our last empirical approach is based on an Exponentially Weighted Moving Average (EWMA) filter. EWMA filters are widely used in practice as a simple and easy-to-implement procedure to accommodate time-varying variances and covariances. EWMA filters have traditionally been based on daily or lower frequency data, but they are equally applicable in the high-frequency realized volatility setting (see, e.g., Fleming, Kirby, and Ostdiek, 2003, for an early application of an EWMA filter with realized covariances).

Let $v_{t}=$ vech $V_{t}$ denote the vectorized daily covariance matrix of interest. The standard EWMA filter based on high-frequency realized covariances may then be expressed as,

$$
v_{t}=(1-\alpha) v_{t-1}+\alpha s_{t-1}
$$

where $\alpha$ defines the decay rate. ${ }^{12}$ Standard choices for $\alpha$ are 0.03 and 0.06 . When $\alpha$ is low the filter is persistent and the filtered $V_{t}$ covariance matrices are fairly stable. When $\alpha$ is high more of the information in $s_{t-1}$ is immediately incorporated into the filtered $V_{t}$. In the analysis reported on below, we estimate $\alpha$ based on the auxiliary assumption that the daily returns are conditionally normally distributed using the same composite likelihood approach described in Appendix C used in estimating the HEAVY $(\mathrm{Q})$ models.

\footnotetext{
${ }^{11}$ Formally, let $\Lambda_{\Sigma}$ and $\Lambda_{V}$ denote the unconditional expectations of the high-frequency and low-frequency daily covariance matrices, respectively, with $\lambda_{\Sigma}$ and $\lambda_{V}$ their vectorized versions. The adjustment coefficient is then defined by $\kappa \equiv L_{N}(\bar{\kappa} \otimes \bar{\kappa}) D_{N}$, where $\bar{\kappa}=\Lambda_{\Sigma}^{1 / 2} \Lambda_{V}^{-1 / 2}$, and $L_{N}$ and $D_{N}$ denote the elimination and duplication matrices respectively (see, e.g., Magnus and Neudecker, 1980).

${ }^{12} \mathrm{As}$ long as the initial $V_{0}$ matrix is positive definite, the EWMA filter automatically ensures that the filtered $V_{t}$ matrices are all positive definite.
} 
In parallel to the models described above, the EWMA filter is readily adapted to incorporate the effect of measurement errors in $s_{t}$ by allowing the $\alpha$ parameter to vary with $\pi_{t}$. Specifically, we define the EWMAQ filter as,

$$
\begin{aligned}
v_{t} & =\left(1-\alpha_{t}\right) \circ v_{t-1}+\alpha_{t} \circ s_{t-1}, \\
\alpha_{t} & =\alpha \iota+\alpha_{Q} \pi_{t-1} .
\end{aligned}
$$

We would naturally expect the $\alpha$ parameter in the EWMAQ filter to be higher than the $\alpha$ parameter in the conventional EWMA filter, allowing for more immediate reactions to $s_{t-1}$ on average. Correspondingly, the $\alpha_{Q}$ parameter should be negative so that the impact of highly uncertain $s_{t-1}$ estimates are attenuated, shifting more of the weight towards the the past filtered $v_{t-1}$ estimates.

\section{Data}

Our empirical analysis is based on a set of ten Dow Jones stocks. The names and ticker symbols for each of the stocks are listed in Table 1. In addition, we use the SPY exchange traded fund in the construct of $\mathrm{S} \& \mathrm{P} 500$ tracking portfolios. We rely on 5-minute returns retrieved from the TAQ database over the February 1993 to December 2013 sample period, for a total of 5,267 daily observations. To most directly highlight the benefits of the high-frequency-based procedures and the new dynamic attenuation models, we focus our analysis on the intraday realized covariances and corresponding open-to-close returns. This also mirrors a number of studies in the recent literature (see, e.g., Lunde, Shephard, and Sheppard, 2015; Hautsch, Kyj, and Malec, 2015; De Lira Salvatierra and Patton, 2015, among others). ${ }^{13}$

Table 1 provides summary statistics for the resulting daily MK covariance estimates. The first two columns report the averages and time series standard deviations of the realized variances for each of the individual asset. The following two columns report the mean and standard deviation of estimated measured errors, based on realized quarticity. The presence of variation in measurement error is the basis for all of the analysis in this paper, and we see from Table 1 that it is substantial: the coefficient of variation is well above one for all ten of these stocks. The last four columns summarize the linear dependence between returns on these assets, showing the average realized correlation of each asset with all other assets, as well as the average realized beta (defined as in Andersen, Bollerslev, Diebold, and Wu, 2006) with respect to the SPY market portfolio. The average correlation among the assets is around 0.28 , indicating important diversification benefits and potentially large gains from risk minimizing portfolios constructed on the basis of more accurate

\footnotetext{
${ }^{13}$ Following Andersen, Bollerslev, and Huang (2011), who treat the overnight returns as "jumps," a separate model could be used to predict the overnight variation. However, a dynamic attenuation model is unlikely to provide any benefits in that context, as the variation of the overnight measurement errors cannot be accurately estimated. Alternatively, following Hansen and Lunde (2005) the intraday variation may be scaled up to reflect the variation for the whole day, akin to the $\kappa$ adjustment term in the HEAVY model.
} 
Table 1: Descriptive Statistics

\begin{tabular}{llllllllll}
\hline Name & Ticker & \multicolumn{2}{c}{ Variance } & \multicolumn{2}{c}{ Quarticity } & \multicolumn{2}{c}{ Correlations } & \multicolumn{2}{c}{ Beta } \\
\hline & & Mean & StDev & Mean & StDev & Mean & StDev & Mean & StDev \\
Market & SPY & 0.954 & 1.165 & 3.882 & 13.933 & & & & \\
American Express & AXP & 3.906 & 55.941 & 3.694 & 12.797 & 0.315 & 1.120 & 1.043 & 0.634 \\
Boeing & BA & 2.782 & 16.425 & 1.714 & 5.192 & 0.271 & 0.934 & 0.821 & 0.584 \\
Chevron & CVX & 1.975 & 13.185 & 0.671 & 1.775 & 0.307 & 1.113 & 0.764 & 0.530 \\
DuPont & DD & 2.723 & 15.397 & 1.562 & 4.466 & 0.315 & 1.132 & 0.928 & 0.562 \\
General Electric & GE & 2.709 & 35.373 & 1.621 & 6.342 & 0.351 & 1.287 & 0.998 & 0.499 \\
IBM & IBM & 2.250 & 10.700 & 1.152 & 3.367 & 0.321 & 1.148 & 0.883 & 0.497 \\
JPMorgan Chase & JPM & 4.329 & 84.575 & 4.392 & 16.741 & 0.316 & 1.110 & 1.172 & 0.656 \\
Coca-Cola & KO & 1.740 & 6.757 & 0.605 & 1.797 & 0.274 & 0.922 & 0.640 & 0.447 \\
Microsoft & MSFT & 2.897 & 13.411 & 1.716 & 4.344 & 0.310 & 1.079 & 1.061 & 0.603 \\
ExxonMobil & XOM & 1.789 & 13.271 & 0.546 & 1.623 & 0.329 & 1.208 & 0.773 & 0.474 \\
\hline
\end{tabular}

Note: The table reports averages and time series standard deviations for the variances of each individual asset, as well as their estimated measurement error variances (Quarticity). Next it depicts averages and standard deviations of the average correlations with the other assets and the final two columns show averages and standard deviations of the realized betas with respect to the SPY market index.

covariance matrix forecasts. The average realized beta equals 0.91. However, the betas vary importantly both across stocks and time, again suggesting potentially large gains from portfolios designed to track the market based on more accurate covariance matrix forecasts. We turn next to a discussion of the models that we rely on below to explore these conjectures.

\section{Model estimates and covariance forecasts}

We begin our empirical analysis by comparing and contrasting the estimated dynamic attenuation models with their constant counterparts based on conventional statistical criteria. Section 5.1 discusses the in-sample parameter estimates and quality of the model fits, while Section 5.2 presents the results from out-of-sample forecast comparisons and corresponding model confidence sets.

\subsection{In-sample estimates}

The parameter estimates obtained for each of the different models are reported in Table 2, along with robust standard errors in parentheses. To conserve space, for the HAR(Q)-DRD models, we only report the averages of the parameter estimates and standard errors over each of the ten individually estimated $\operatorname{HAR}(\mathrm{Q})$ variance models.

As expected, all of the estimated Q-coefficients are negative and statistically significant. This directly corroborates the basic idea and mechanics of the models, that as the measurement error variance $\pi_{t}$ increases (decreases), the informativeness of the past covariance estimate decreases (increases), resulting in the models endogenously increasing (decreasing) the degree of attenuation. To put the magnitude of the attenuation in further perspective, the cross-sectional average of the 
Table 2: In-Sample Estimates

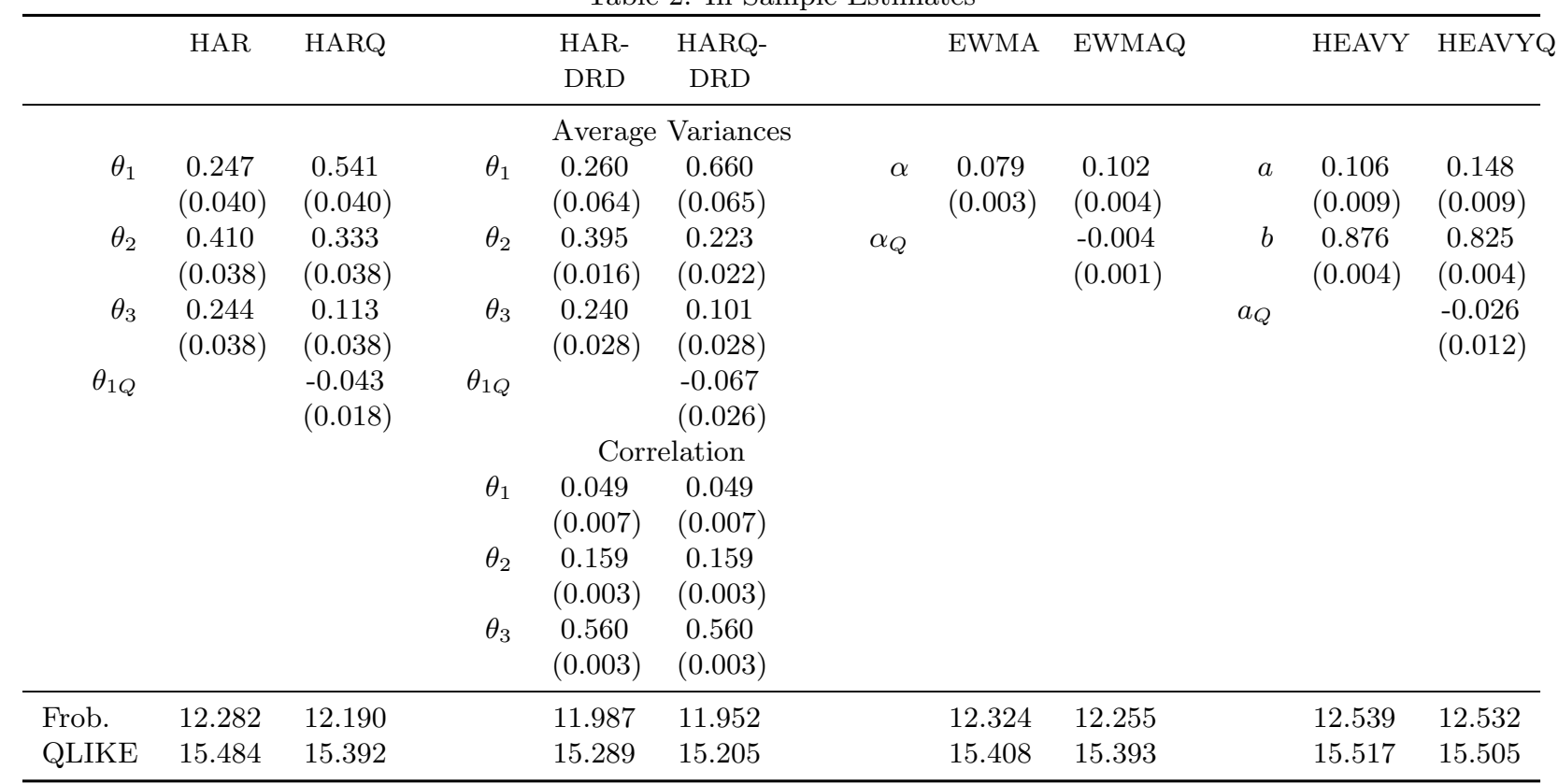

Note: The table reports in-sample parameter estimates and measures of fit for the different models. For the $\operatorname{HAR}(\mathrm{Q})$-DRD models the reported parameter estimates and standard errors for the variance specification are the averages across the ten individually estimated univariate HAR(Q) models. The bottom panel reports the in-sample fit for the different models as measured by the Frobenius distance and the QLIKE loss.

composite $\theta_{1, t}$ parameter for the HARQ model varies substantially over the sample, from a low of 0.21 to a high of 0.87 . Correspondingly, there is a "redistribution" of weight from the longer weekly and monthly lags to the daily lag when comparing the HARQ to HAR model. This effect is even more pronounced for the $\operatorname{HAR}-\mathrm{DRD}(\mathrm{Q})$ models, in which the individual variances have their own separate dynamics, and in turn their own individual sensitivity to the degree of measurement error. As such, the (average) estimated $\theta_{1 Q}$ parameter is also greater (in absolute value) for the HARQ-DRD model than it is for the HARQ model. ${ }^{14}$

The same results carry over to the EWMA(Q) filters and HEAVY(Q) models. The estimated $\alpha$ for the EWMAQ filter exceeds the $\alpha$ for the standard EWMA filter, allowing for a greater immediate impact of the realized covariance on average. However, when the estimation error is large, the negative $\alpha_{Q}$ implies that the weight is shifted away from the current noisy estimate towards the long-run weighted average. Similarly, the estimated a parameter is higher for the HEAVYQ model than for the standard HEAVY model, together with a negative $a_{Q}$ estimate for the HEAVYQ model.

In addition to the parameter estimates, the last two rows in Table 2 report the Frobenius distance and QLIKE loss for the fitted covariance matrices, say $H_{t}$, with respect to the ex-post

\footnotetext{
${ }^{14}$ This in itself represents an attenuation effect, as the measurement error in $\pi_{t}$ aggregates in the cross-section for the HARQ model, rendering the dynamic $\theta_{1 Q}$ parameter less effective.
} 
realized covariances $S_{t}$. The Frobenius norm is commonly used to measure the distance between two matrices,

$$
L_{t}^{\text {Frobenius }}=\sqrt{\operatorname{Tr}\left(H_{t}-S_{t}\right)}
$$

The quasi-likelihood (QLIKE) measure is based on the negative of the log-likelihood of a multivariate normal,

$$
L_{t}^{Q L I K E}=\log \left|H_{t}\right|+\operatorname{Tr}\left(H_{t}^{-1} S_{t}\right)
$$

The results reported in the table are obtained by summing $L_{t}^{\text {Frobenius }}$ and $L_{t}^{Q L I K E}$ over the full sample. Both functions measure "loss," so that lower values are preferable. ${ }^{15}$ With the exception of the QLIKE loss for the EWMAQ filter, the dynamic attenuation models systematically result in lower losses than their constant counterparts. The HARQ-DRD model results in the lowest in-sample loss overall. The next section investigates how the models compare on an out-of-sample basis using these same statistical loss functions.

\subsection{Out-of-sample forecasts}

Our out-of-sample forecast comparisons are based on the one-day-ahead forecasts for the same ten-dimensional covariance matrix and set of models analyzed above. All of the models are reestimated every day based on a rolling sample window of the past 1,000 days. ${ }^{16}$ The forecasts are evaluated based on the same Frobenius distance and QLIKE loss defined in equations (13) and (14), respectively, where the in-sample fitted value $H_{t}$ are replaced with the forecast from the relevant model.

Consistent with the in-sample results, the full out-of-sample results reported in the first two columns of Table 3 show that our new dynamic attenuation models systematically improve on their non-attenuated counterparts. If anything, the out-of-sample improvements are even bigger. This holds true for both loss functions. ${ }^{17}$ To help understand where these forecast improvements are coming from, the last four columns of the table split the sample into days with the $95 \%$ lowest and $5 \%$ highest measurement error variances, respectively, as defined by the Frobenius norm of $\pi_{t}$. As the table shows, the dynamic attenuation models offers improvements in both situations, by either increasing the responsiveness when the realized covariances are precisely estimated, or increasing the attenuation when the estimates are poor. Interestingly, however, the largest percentage improvements stem from the increased responsiveness when the estimates are precise.

\footnotetext{
${ }^{15}$ Despite the use of an ex-post estimate in place of the true covariance matrix, both of the loss functions provide consistent model rankings, as further discussed in Patton (2011) and Laurent, Rombouts, and Violante (2013).

${ }^{16}$ Due to the difficulties in estimating the integrated quarticity matrix, the HARQ and HARQ-DRD models occasionally produce negative definite forecasts. If this occurs, we apply an "insanity filter," and replace the negative definite covariance matrix forecast with the simple average realized covariance over the relevant estimation sample. This only happens for one or two forecasts per series over the entire sample.

${ }^{17}$ Unreported results show that all of the models produce approximately unbiased forecasts, and that the apparent improvements for the Q-models mainly stem from a reduction in the forecast error variance.
} 
Table 3: Out-of-Sample Forecast Results

\begin{tabular}{|c|c|c|c|c|c|c|}
\hline & \multicolumn{2}{|c|}{ Full Sample } & \multicolumn{2}{|c|}{ Lower $95 \%\left\|\pi_{t}\right\|$} & \multicolumn{2}{|c|}{ Upper $5 \%\left\|\pi_{t}\right\|$} \\
\hline & Frob. & QLIKE & Frob. & QLIKE & Frob. & QLIKE \\
\hline HAR & 12.305 & 14.382 & 9.274 & 13.275 & 69.723 & 32.651 \\
\hline HARQ & $12.107^{*}$ & $14.159^{*}$ & $9.161^{*}$ & $13.206^{*}$ & 69.703 & $32.190 *$ \\
\hline HAR-DRD & 12.134 & 14.140 & 8.940 & 13.022 & 70.467 & 32.603 \\
\hline HARQ-DRD & $11.976^{*}$ & $13.896^{*}$ & $8.888^{*}$ & $12.990^{*}$ & $70.056^{*}$ & $31.050 *$ \\
\hline EWMA & 12.379 & 14.105 & 9.303 & 13.109 & 71.104 & 32.686 \\
\hline EWMAQ & $12.178^{*}$ & $14.091^{*}$ & $9.172^{*}$ & 13.122 & $70.633^{*}$ & $32.643^{*}$ \\
\hline HEAVY & 12.473 & 14.051 & 9.299 & 13.041 & 71.789 & 32.263 \\
\hline HEAYVQ & $12.161^{*}$ & $14.004^{*}$ & $9.093^{*}$ & 13.050 & $70.170^{*}$ & 32.258 \\
\hline
\end{tabular}

Note: The table reports out-of-sample forecast loss for the different models. The first two columns are based on the full-sample. The last four columns split the sample into days when the measurement error variance is low, and $\left\|\pi_{t}\right\|$ is below the $95 \%$ quantile, and days when the estimation error is high, and $\left\|\pi_{t}\right\|$ is above the $5 \%$ quantile. Entries in boldface indicate models that are part of the $90 \%$ model confidence set (MCS) for the relevant column. Q-models that significantly improve on their non-Q benchmark are indicated by an asterisk.

In order to formally determine whether the quality of the forecasts differ significantly across the different models, we apply the Model Confidence Set (MCS) of Hansen, Lunde, and Nason (2011). This approach identifies the (sub)set of models that contains the best forecasting model with $90 \%$ confidence. For each of the two loss functions and three sample-splits we determine the subset of models that comprise the MCS (members of the set indicated in boldface). Additionally, we separately compare each of the Q-models with their non-attenuated counterpart using the Diebold and Mariano (2002) test (significance indicated by *).

Looking first at the pairwise comparisons, the Diebold and Mariano (2002) test confirms that each of the Q-models significantly outperforms their respective benchmark over the full sample. This is true irrespective of the loss function used in comparing the models. The Q-models generally also beat their non-Q counterparts over each of the two split samples, but the differences are not always significant. Jointly comparing all of the eight models, the HARQ-DRD model stands out as always being included in the MCS, regardless of the loss function and/or sample-split underlying the comparisons. The EWMAQ filter and HEAVYQ model are also both included the MCS for the full sample based on either loss function. The HARQ model, on the other hand, appears to work particularly well when the covariances are imprecisely estimated. Taken as whole, the results clearly corroborate the superior statistical performance of the dynamic attenuation models.

The next section seeks to address whether these statistical improvements translate into economic gains by evaluating the new dynamic attenuation models from a practical portfolio allocation perspective.

\section{Minimum variance and tracking error portfolios}

Our economic evaluations of the different models are based on their use in the construction of Global Minimum Variance (GMV) portfolios and portfolios designed to track the aggregate market. 
The GMV and tracking portfolio weights only depend on return covariances, and as such provide an especially clean framework for assessing the merits of the different covariance forecasting models. Most other portfolio allocation decisions depend on forecasts for the expected returns as well, and these are notoriously difficult to accurately estimate. As reported by Jagannathan and Ma (2003) and DeMiguel, Garlappi, Nogales, and Uppal (2009a), mean-variance optimized portfolios typically do not perform as well as GMV portfolios in terms of out-of-sample Sharpe ratios, as the estimation error in the expected returns tend to distort the positions. In order to most directly highlight the advantages of the new dynamic attenuation models, we initially focus on a daily investment horizon. Daily re-balancing schemes have also previously been employed in a number of studies concerned with volatility-timing strategies (see, e.g., Fleming, Kirby, and Ostdiek, 2003; Fan, Li, and Yu, 2012, among others). In Section 8, we also consider weekly and monthly investment horizons.

\subsection{Practical implementation and utility comparisons}

Consider a risk-averse investor who allocates her funds into $N$ risky assets based on the forecasts for the daily covariance matrix of the returns on the assets, $H_{t \mid t-1}$. To minimize the conditional volatility, the investor solves the global minimum variance portfolio problem,

$$
\begin{gathered}
w_{t}=\arg \min w_{t}^{\prime} H_{t \mid t-1} w_{t} \\
\text { s.t. } \quad w_{t}^{\prime} \iota=1,
\end{gathered}
$$

where $\iota$ is a $N \times 1$ vector of ones, resulting in the optimal portfolio allocation vector,

$$
w_{t}=\frac{H_{t \mid t-1}^{-1} \iota}{\iota^{\prime} H_{t \mid t-1}^{-1} \iota} .
$$

In the sequel, we will denote the $n$th element of $w_{t}$, corresponding to the allocation to the $n$th asset, by $w_{t}^{(n)}$. Correspondingly, we will denote the return on the $n$th asset by $r_{t}^{(n)}$.

One potentially important feature of the dynamic attenuation models is that they result in more stable covariance matrix forecasts, and therefore less turnover than conventional procedures. Importantly, this should make trading strategies based on the new models cheaper to implement. To evaluate this, we assume that the investor faces fixed transaction $\operatorname{costs} c$ proportional to the turnover rates in her portfolios. Specifically, following standard arguments (see, e.g., the discussion in Han, 2006; Liu, 2009; DeMiguel, Nogales, and Uppal, 2014), the total portfolio turnover from day $t$ to day $t+1$ is readily measured by,

$$
T O_{t}=\sum_{n=1}^{N}\left|w_{t+1}^{(n)}-w_{t}^{(n)} \frac{1+r_{t}^{(n)}}{1+w_{t}^{\prime} r_{t}}\right| .
$$

With proportional transaction $\operatorname{costs} c T O_{t}$, the portfolio excess return net of transaction cost is 
therefore,

$$
r_{p t}=w_{t}^{\prime} r_{t}-c T O_{t}
$$

In the results below, we consider values of $c$ ranging from 0 to $2 \%$, in line with values for this parameter employed in earlier studies, see Fleming et al. (2003) and Brown and Smith (2011) for example.

The more stable and less susceptible to estimation error covariance matrix forecasts from the dynamic attenuation models should also result in less extreme portfolio allocations. To assess this, we also report the portfolio concentrations,

$$
C O_{t}=\left(\sum_{n=1}^{N} w_{t}^{(n) 2}\right)^{1 / 2}
$$

and the total portfolio short positions,

$$
S P_{t}=\sum_{n=1}^{N} w_{t}^{(n)} \mathbb{I}_{\left\{w_{t}^{(n)}<0\right\}} .
$$

Again, less extreme and fewer short positions are likely to facilitate the practical implementation of the portfolios, and help further mitigate transaction costs in situations when the costs are not simply proportional to the turnover.

In addition to the GMV portfolios define in (16), we also consider portfolios designed to track the SPY market portfolio. Many institutional investors are evaluated based on their performance relative to a benchmark, thus the ability to closely track a particular portfolio is often of great import. Our construction of the tracking portfolios is based on minimizing the variance of the tracking error, or equivalently the GMV for the returns on each of the assets in excess of the SPY return. Since the assets correlate to varying degrees with the market, the covariances among the returns net of the market return are typically more dispersed than the covariances of the returns themselves. As such, this renders the construction of the minimum tracking error portfolios more challenging and potentially also more prone to extreme positions than the GMV portfolios.

To evaluate the economic significance of the different forecasting models, we consider the utilitybased framework of Fleming, Kirby, and Ostdiek (2001, 2003). In particular, assuming that the investor has quadratic utility with risk aversion $\gamma$, the realized daily utility generated by the portfolio based on the covariance forecasts from model $k$, may be expressed as

$$
U\left(r_{p t}^{k}, \gamma\right)=\left(1+r_{p t}^{k}\right)-\frac{\gamma}{2(1+\gamma)}\left(1+r_{p t}^{k}\right)^{2}
$$


The economic value of the different models may therefore be determined by solving for $\Delta_{\gamma}$ in

$$
\sum_{t=1}^{T} U\left(r_{p t}^{k}, \gamma\right)=\sum_{t=1}^{T} U\left(r_{p t}^{l}-\Delta_{\gamma}, \gamma\right)
$$

where $\Delta_{\gamma}$ can be interpreted as the return an investor with risk aversion $\gamma$ would be willing to sacrifice to switch from using model $k$ to using model $l$. In order to determine whether the $\Delta_{\gamma}$ are significantly different from zero we use the Reality Check of White (2000), based on the stationary bootstrap of Politis and Romano (1994), with 999 bootstrap samples and an average block length of 22 days.

\section{Dynamic attenuation models in action}

In parallel to the statistical model comparisons in Section 5.2, in our economic evaluations we rely on a one-day-ahead out-of-sample forecasting scheme, in which we re-estimate all of the models based on a rolling estimation window of 1,000 days, re-balancing the resulting portfolios daily. In addition to the basic features of the daily GMV and tracking error portfolios, we are particularly interested in whether accurate and stable covariance matrix forecasts from the dynamic attenuation models manifest in systematically lower portfolio turnover and reduced transaction costs. We begin by considering the results from an empirically realistic simulation study explicitly designed to highlight these features. We then present our main empirical findings.

\subsection{Fundamental and spurious turnover}

The statistically more accurate covariance forecasts from the dynamic attenuation models should result in portfolio allocations closer to the optimal weights implied by the true covariance matrix. The more stable forecasts from the attenuation models should also result in more stable portfolio allocations and a reduction in turnover. Comparing the estimated GMV portfolios with the optimal GMV portfolio based on the true covariance matrix within a controlled simulation setting, allow us to directly asses these conjectures and dissect the workings of the models.

To keep the simulations manageable, we restrict the analysis to $N=5$ and focus on the easy-toimplement vech HAR and HARQ models. We begin by simulating 2,000 days of one-second returns; a more detailed description of the simulation setup is given in Appendix B. We then aggregate the one-second returns to 1,5 and 15 -minute returns $(M=390,78,26)$, and estimate the models on rolling windows of the corresponding 1,000 daily MK estimates, resulting in a total of 1,000 out-ofsample portfolio decisions for each of the different models and procedures. As a baseline, we also consider a HAR model for the true population covariances $\Sigma_{t}$, so that there are no measurement errors, only forecast errors. We will refer to this model as $\mathrm{HAR}_{\infty}$. We report the portfolio turnover defined in (17), as well as the portfolio standard deviation based on the true covariances, $\sqrt{\hat{w}_{t} \Sigma_{t} \hat{w}_{t}^{\prime}}$. To asses how far the estimated weights are from the "fundamental" weights, we also report the 
Table 4: Simulation Results: Fundamental and Spurious Turnover

\begin{tabular}{|c|c|c|c|c|c|c|c|c|}
\hline \multirow[t]{2}{*}{ M } & \multicolumn{2}{|c|}{$\infty$} & \multicolumn{2}{|c|}{390} & \multicolumn{2}{|c|}{78} & \multicolumn{2}{|c|}{26} \\
\hline & Optimal & HAR & HAR & HARQ & HAR & HARQ & HAR & HARQ \\
\hline Turnover & 0.2206 & 0.1878 & 0.2379 & 0.2071 & 0.2830 & 0.2257 & 0.2999 & 0.2360 \\
\hline StDev & 0.1650 & 0.1829 & 0.1844 & 0.1837 & 0.1879 & 0.1853 & 0.1920 & 0.1887 \\
\hline Distance to Optimal & 0.0000 & 0.0446 & 0.0471 & 0.0457 & 0.0531 & 0.0488 & 0.0601 & 0.0457 \\
\hline Distance to $\mathrm{HAR}_{\infty}$ & 0.0446 & 0.0000 & 0.0104 & 0.0062 & 0.0198 & 0.0102 & 0.0231 & 0.0141 \\
\hline
\end{tabular}

Note: The table reports the results from a simulation study pertaining to the out-of-sample GMV portfolios. The covariance matrix forecasts are based on the HAR and HARQ models and the MK estimates with $M$ intraday observations. The $M=\infty$ column shows the results for the HAR model applied to population covariance matrix as well as the optimal results based on ex-post estimates. Turnover is defined in equation (17). StDev refers to the portfolio standard deviation based on the population covariance. Distance reports the distance, as defined in the main text, between the portfolio weights and the weights based on the true covariance matrix $\Sigma_{t}$, or the weights based on the $H A R_{\infty}$ model.

distance to the weights based on the true covariances, $\sqrt{\sum_{n}\left(\hat{w}_{t}^{(n)}-w_{t}^{(n)}\right)^{2}}$. Finally, we report the distance of the portfolio weights to the weights implied by the $\mathrm{HAR}_{\infty}$ model based on the true integrated covariances.

Table 4 details the findings from the simulations. To begin, note that even if the true covariances are observed, there are still non-trivial forecast errors, as evidenced by the results for the $\mathrm{HAR}_{\infty}$ model. This provides a useful benchmark for the other models. Comparing the results for the HAR models across the different sampling frequencies clearly shows the detrimental impact of unreliable realized covariances, as manifest in higher turnover, portfolio standard deviation, and distance to the true $\Sigma_{t}$ weights for lower values of $M$ and increasingly inaccurate realized covariance matrices. By contrast, the performance of the HARQ model is much more stable across the different values of $M$, and also closer to the $\mathrm{HAR}_{\infty}$ benchmark model. Compared to the HAR model, the HARQ model reduces the spurious turnover induced by estimation error by more than half when $M=26$, and even more for $M=78$ and $M=390 .{ }^{18}$ In addition to the reduced excess turnover, the HARQ portfolios also achieve lower ex-post standard deviations than the HAR portfolios, and portfolio weights that are systematically closer to the true $\Sigma_{t}$ weights.

\subsection{Empirical intuition}

Before presenting our main empirical results, it is instructive to visualize the key features of the dynamic attenuation models behind our findings. To do so, we consider a simple illustrative example based on just two stocks, Boeing (BA) and JP Morgan (JPM), over the two-week period from August 11-25, 2002. Following the simulations discussed in the previous section, we focus on the vech HAR and HARQ models, and the use of these models in the construction of daily GMV portfolios.

\footnotetext{
${ }^{18}$ The greater improvements relative to the HAR model for higher $M$ stem from the fact that the degree of measurement error also needs to be estimated, which similarly becomes more accurate for higher values of $M$.
} 
Figure 1: Dynamic Shrinkage
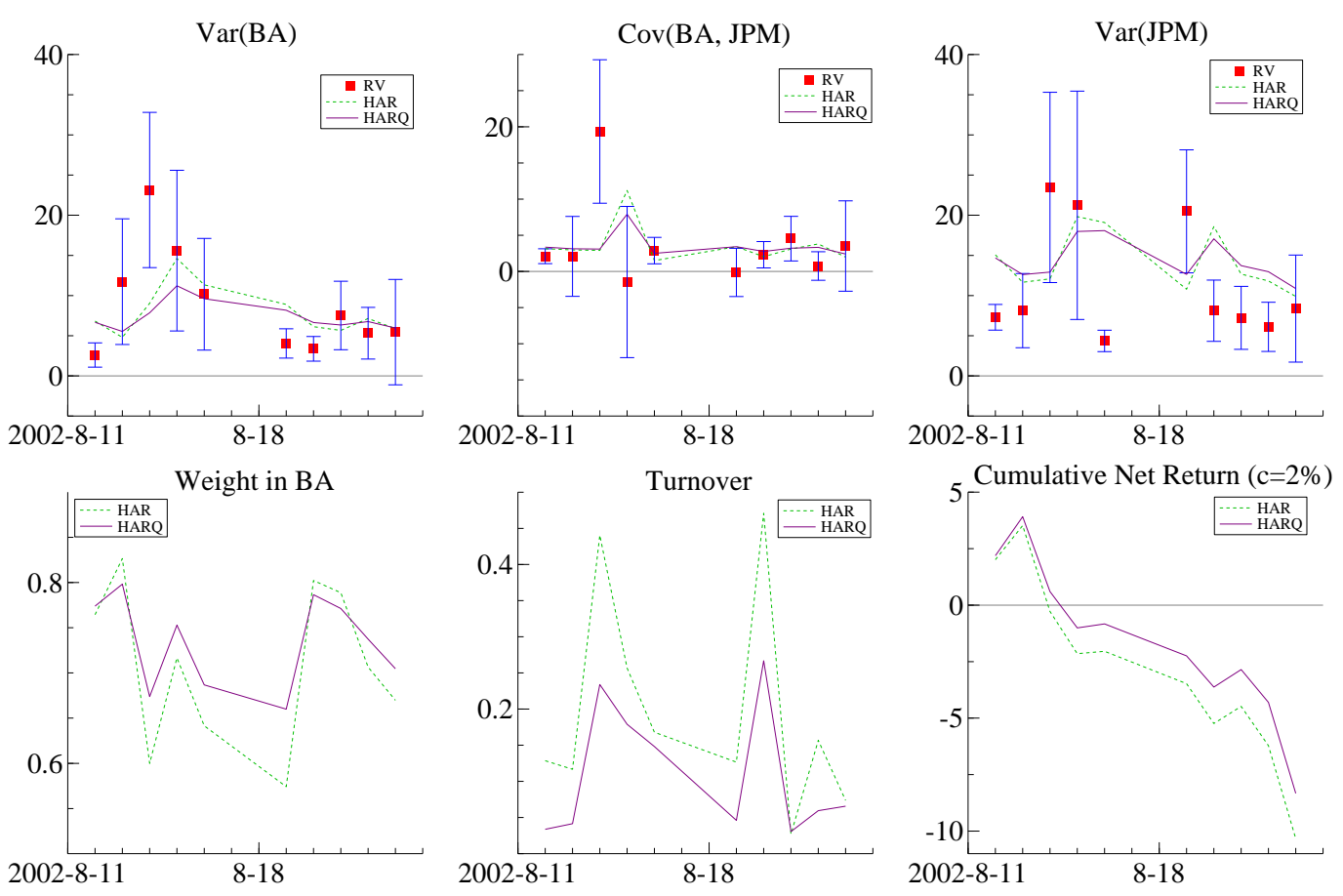

Note: The top row of panels shows the estimated (co)variance for BA and JPM, along with the forecasted values from the HAR and HARQ models. The bottom left panel shows the GMV weights for BA based on the HAR and HARQ models, the middle panel shows the turnover, and the right panel the cumulative portfolio return net of transaction costs.

The top row in Figure 1 shows the ex-post estimated realized (co)variances and corresponding 95\% confidence bounds, along with the one-day-ahead HAR and HARQ model forecasts. Evidently, the HARQ forecasts respond less aggressively to the lagged (co)variances than the HAR-based forecasts when the measurement errors are large. Since large uncertainty is often accompanied by transitory spikes in volatility, the HARQ-based forecasts appear more stable over time. Importantly, this translates into more stable portfolio allocations, as evidenced by the first two panels in the bottom row, which display the portfolio weight for BA and the portfolio turnover. Although the allocations for both of the models average around $70 \%$ to BA, the variability around this weight is obviously lower for the HARQ model. This in turn results in uniformly lower turnover for the HARQ model, and a reduction in transaction costs, as seen in the bottom right panel, which displays the cumulative net return over the two-week period based on transaction costs $c=2 \%$. While these two-week cumulative differences may appear numerically small, the results in the next section show that they add up to substantial improvements on an annual basis. 
Table 5: Unconstrained Global Minimum Variance Portfolio Allocations

\begin{tabular}{|c|c|c|c|c|c|c|c|c|c|}
\hline & & HAR & HARQ & $\begin{array}{l}\text { HAR- } \\
\text { DRD }\end{array}$ & $\begin{array}{l}\text { HARQ- } \\
\text { DRD }\end{array}$ & EWMA & EWMAQ & HEAVY & HEAVYQ \\
\hline TO & & 0.522 & 0.385 & 0.391 & 0.339 & 0.135 & 0.096 & 0.172 & 0.122 \\
\hline $\mathrm{CO}$ & & 0.513 & 0.517 & 0.487 & 0.497 & 0.505 & 0.506 & 0.497 & 0.497 \\
\hline $\mathrm{SP}$ & & -0.105 & -0.109 & -0.070 & -0.082 & -0.095 & -0.096 & -0.089 & -0.089 \\
\hline Mean Ret & & 3.079 & 3.156 & 3.612 & 4.371 & 3.455 & 3.867 & 3.847 & 4.124 \\
\hline StDev Ret & & 14.912 & 14.781 & 15.016 & 14.566 & 14.972 & 14.637 & 14.922 & 14.614 \\
\hline \multirow[t]{3}{*}{$c=0 \%$} & Sharpe & 0.206 & 0.214 & 0.241 & 0.300 & 0.231 & 0.264 & 0.258 & 0.282 \\
\hline & $\Delta_{1}$ & & 9.7 & & $82.6^{*}$ & & 46.2 & & 32.2 \\
\hline & $\Delta_{10}$ & & 27.3 & & $142.6^{*}$ & & $90.9^{*}$ & & $73.3^{*}$ \\
\hline \multirow[t]{3}{*}{$c=1 \%$} & Sharpe & 0.118 & 0.148 & 0.175 & 0.241 & 0.208 & 0.248 & 0.229 & 0.261 \\
\hline & $\Delta_{1}$ & & $44.3^{*}$ & & $95.6^{*}$ & & $55.9^{*}$ & & 44.9 \\
\hline & $\Delta_{10}$ & & $61.9^{*}$ & & $155.6^{*}$ & & $100.7^{*}$ & & $86.0^{*}$ \\
\hline \multirow[t]{3}{*}{$c=2 \%$} & Sharpe & 0.030 & 0.082 & 0.109 & 0.183 & 0.185 & 0.231 & 0.200 & 0.240 \\
\hline & $\Delta_{1}$ & & $78.8^{*}$ & & $108.6^{*}$ & & $65.7^{*}$ & & $57.6^{*}$ \\
\hline & $\Delta_{10}$ & & $96.4^{*}$ & & $168.7^{*}$ & & $110.4^{*}$ & & $98.7^{*}$ \\
\hline
\end{tabular}

Note: The table shows the results for the global minimum variance portfolio (GMV). We report turnover (TO), portfolio concentration (CO), and short positions (SP), as well as the average annualized return and standard deviation. Standard deviations in bold indicate the models that belong to the $90 \%$ model confidence set (MCS) of lowest ex-post daily volatility. The table also reports the economic gains of switching from the conventional model to the Q-model in annual basis points, $\Delta_{\gamma}$, for various transaction cost levels $c$ and risk aversion coefficients $\gamma$. Asterisks denote $\Delta_{\gamma}$ significantly different from zero at the $5 \%$ level.

Table 6: Unconstrained Minimum Tracking Error Portfolio Allocations

\begin{tabular}{lccccrrrrr}
\hline & HAR & HARQ & $\begin{array}{l}\text { HAR- } \\
\text { DRD }\end{array}$ & $\begin{array}{l}\text { HARQ- } \\
\text { DRD }\end{array}$ & EWMA & EWMAQ & HEAVY & HEAVYQ \\
\hline TO & & 0.173 & 0.102 & 0.134 & 0.114 & 0.063 & 0.045 & 0.080 & 0.057 \\
CO & 0.339 & 0.338 & 0.339 & 0.342 & 0.340 & 0.340 & 0.338 & 0.338 \\
SP & & -0.000 & -0.000 & -0.000 & -0.000 & -0.000 & -0.000 & -0.000 & -0.000 \\
StDev TE & 6.618 & $\mathbf{6 . 4 8 9}$ & 6.610 & $\mathbf{6 . 5 2 1}$ & 6.636 & $\mathbf{6 . 5 1 3}$ & 6.637 & $\mathbf{6 . 5 0 6}$ \\
\hline \multirow{2}{*}{$c=0 \%$} & $\Delta_{1}$ & & 29.1 & & 27.8 & & 33.6 & & 32.5 \\
& $\Delta_{10}$ & & 39.1 & & $35.0^{*}$ & & 43.4 & & 42.8 \\
$c=1 \%$ & $\Delta_{1}$ & & $46.9^{*}$ & & $42.8^{*}$ & & 38.2 & & 38.5 \\
& $\Delta_{10}$ & & $56.9^{*}$ & & $50.0^{*}$ & & $47.9^{*}$ & & 48.8 \\
& $\Delta_{1}$ & & $64.6^{*}$ & & $47.8^{*}$ & & $42.7^{*}$ & & $44.5^{*}$ \\
& $\Delta_{10}$ & & $74.6^{*}$ & & $55.0^{*}$ & & $52.5^{*}$ & & $54.8^{*}$ \\
\hline
\end{tabular}

Note: The table shows the results for the the minimum tracking error portfolio. We report turnover (TO), portfolio concentration (CO), and short positions (SP), as well as the standard deviation of the tracking error (TE). Standard deviations in bold indicate the models that belong to the $90 \%$ model confidence set (MCS) of lowest ex-post daily volatility. The table also reports the economic gains of switching from the conventional model to the Q-model in annual basis points, $\Delta_{\gamma}$, for various transaction cost levels $c$ and risk aversion coefficients $\gamma$. Asterisks denote $\Delta_{\gamma}$ significantly different from zero at the $5 \%$ level.

\subsection{Dynamically attenuated portfolio allocations}

Our main empirical results are reported in Tables 5 and 6 . The first table gives the results related to the GMV portfolios, while the second reports the results for the portfolios designed to track the market. Looking first at the summary numbers in the top panel of Table 5, the HARQ-DRD model yields the lowest portfolio variance overall, and using the Model Confidence 
Set (MCS) approach of Hansen, Lunde, and Nason (2011), we find the ex-post portfolio variances for the EWMAQ filter and the HEAVYQ model are not significantly larger. All other models generate significantly larger portfolio variances. The variation in the turnover observed across the different classes of models is quite large. Interestingly, however, even in the absence of transaction costs the models with the highest turnover do not result in the highest Sharpe ratios and/or lowest portfolio variance. On the contrary, all of the dynamic attenuation models result in lower turnover than their conventional benchmarks, while attaining a reduction in variance, an increase in average returns, and a higher Sharpe ratio. ${ }^{19}$

The bottom panel of Table 5 shows the economic gains of switching from each of the conventional models to their dynamic attenuation counterparts. In the absence of transaction costs, or $c=0 \%$, the gains range between 10 and 140 basis points annually, depending on the model and coefficient of risk aversion. Since all of the Q-models result in lower turnover, these gains rise to 60 to 170 basis points when the transaction costs rise from $c=0 \%$ to $c=2 \% .{ }^{20}$

Turning to Table 6 and the results for the minimum tracking error portfolios tell a similar story. The standard deviation of the tracking error is significantly reduced by the Q-models, which jointly comprise the model confidence set of minimum TE models. The percentage reductions in turnover also closely mirror those of the GMV portfolios. Meanwhile, as the turnover for the tracking portfolios tend to be somewhat lower than for the GMV portfolios, the economic gains are similarly reduced. Nonetheless, the gains are consistently positive ranging from between 30 to 70 basis points annually. Again, these are non-trivial improvements from a practical investment perspective.

As indicated by the summary statistics in the top part of Table 5, many of the portfolios in Table 5, and the GMV portfolios in particular, involve negative or short positions. Short positions are generally more costly to implement than long positions. Many financial institutions are also explicitly prevented from holding short positions. Hence, in an effort to investigate the sensitivity of our results to the imposition of no-short-sale constraints, we repeat the previous analysis by adding the constraint that $w_{t}^{(n)} \geq 0, \forall n$ to the optimization problem in (15). While this constrained optimization problem no longer allows for an explicit closed form solution to the optimal portfolio weights, the constrained minimum variance portfolios may easily be solved for numerically using standard quadratic programming tools (see, e.g., Lawrence and Tits, 2001).

The results pertaining to these constrained minimum variance portfolios not allowing for shortsales are reported in Table $7 .{ }^{21}$ Interestingly, and consistent with the prior empirical evidence in

\footnotetext{
${ }^{19}$ This finding is in line with the low volatility anomaly documented in Chan, Karceski, and Lakonishok (1999), Jagannathan and Ma (2003) and Baker, Bradley, and Wurgler (2011), among others.

${ }^{20}$ The turnover is reduced by a minimum of $14 \%$ for the HARQ-DRD model up to almost $30 \%$ for the HEAVYQ model.

${ }^{21}$ Since the unconstrained tracking portfolios involve much fewer short positions, the results for the constrained tracking portfolios are much closer to the results in Table 5, and we purposely defer these results to Appendix D to conserve space.
} 
Table 7: No Short-Sale Minimum Variance Portfolios

\begin{tabular}{|c|c|c|c|c|c|c|c|c|c|}
\hline & & HAR & HARQ & $\begin{array}{l}\text { HAR- } \\
\text { DRD }\end{array}$ & $\begin{array}{l}\text { HARQ- } \\
\text { DRD }\end{array}$ & EWMA & EWMAQ & HEAVY & HEAVYQ \\
\hline TO & & 0.390 & 0.284 & 0.322 & 0.280 & 0.101 & 0.071 & 0.131 & 0.093 \\
\hline $\mathrm{CO}$ & & 0.477 & 0.479 & 0.466 & 0.471 & 0.475 & 0.475 & 0.469 & 0.469 \\
\hline Mean Ret & & 3.755 & 3.927 & 3.922 & 4.633 & 3.925 & 4.355 & 4.229 & 4.668 \\
\hline StDev Ret & & 15.365 & 14.977 & 15.296 & 14.855 & 15.260 & 14.821 & 15.249 & 14.784 \\
\hline \multirow[t]{3}{*}{$c=0 \%$} & Sharpe & 0.244 & 0.262 & 0.256 & 0.312 & 0.257 & 0.294 & 0.277 & 0.316 \\
\hline & $\Delta_{1}$ & & 23.1 & & $77.8^{*}$ & & 62.3 & & 64.7 \\
\hline & $\Delta_{10}$ & & 76.1 & & $137.6^{*}$ & & $114.7^{*}$ & & $120.0^{*}$ \\
\hline \multirow[t]{3}{*}{$c=1 \%$} & Sharpe & 0.180 & 0.214 & 0.203 & 0.264 & 0.241 & 0.282 & 0.256 & 0.300 \\
\hline & $\Delta_{1}$ & & 49.7 & & 88.4 & & $57.0^{*}$ & & 60.6 \\
\hline & $\Delta_{10}$ & & $102.8^{*}$ & & $148.3^{*}$ & & $116.4^{*}$ & & $123.6^{*}$ \\
\hline \multirow[t]{3}{*}{$c=2 \%$} & Sharpe & 0.116 & 0.167 & 0.150 & 0.217 & 0.224 & 0.270 & 0.234 & 0.284 \\
\hline & $\Delta_{1}$ & & $76.4^{*}$ & & $99.0 *$ & & $64.3^{*}$ & & $70.3^{*}$ \\
\hline & $\Delta_{10}$ & & $129.4^{*}$ & & $158.9^{*}$ & & $123.9^{*}$ & & $133.2^{*}$ \\
\hline
\end{tabular}

Note: The table reports the results for minimum variance portfolios that do not allow for short positions. The table shows the portfolio turnover (TO), portfolio concentration (CO), together with the average annualized return and standard deviation. Standard deviations in bold indicate the models that belong to the $90 \%$ model confidence set (MCS) of lowest ex-post daily volatility. The table also reports the economic gains of switching from the conventional model to the Q-model in annual basis points, $\Delta_{\gamma}$, for various transaction cost levels $c$ and risk aversion coefficients $\gamma$. Asterisks denote $\Delta_{\gamma}$ significantly different from zero at the $5 \%$ level.

Jagannathan and Ma (2003), the no short-sale restriction generally leads to higher ex-post Sharpe ratios than for the unconstrained GMV portfolios. ${ }^{22}$ The no-short-sale constraint also systematically reduces turnover. As such, the economic gains from the dynamic attenuation models are slightly lower compared to the gains for the unconstrained GMV portfolios in Table 5. Nonetheless, the benefits of switching to the new Q-models remain economically large and statistically significant, ranging up to nearly 160 basis points per year in some situations.

\section{Longer horizons and alternative procedures}

Dynamic portfolio decisions naturally present a tradeoff between the horizon over which the pertinent risks are assumed to be constant versus the accuracy with which the risks can be measured and the costs of implementing the investment decisions. In the results reported above, we relied on a daily horizon for both estimating the new models and re-balancing the portfolios. This section presents additional results pertaining to longer weekly and monthly investment horizons. In addition to the use of coarser re-balancing schemes, alternative shrinkage type procedures have previously been advocated in the literature to help mitigate the impact of estimation errors and excessive turnover. Below we also compare and contrast the new dynamic attenuation procedures developed here with some of these alternative shrinkage type procedures, in which the covariance

\footnotetext{
${ }^{22}$ As discussed by Jagannathan and Ma (2003), restricting the portfolio weights to be non-negative may be seen as a way to limit the impact of estimation errors, akin to the main idea behind the new dynamic attenuation models, and the more traditional shrinkage type procedures discussed further below.
} 
Table 8: Longer Horizon Portfolio Allocations

\begin{tabular}{lllccc}
\hline & & \multicolumn{2}{c}{ Weekly } & \multicolumn{2}{c}{ Monthly } \\
& & HAR-DRD & HARQ-DRD & HAR-DRD & HARQ-DRD \\
\hline TO & 0.112 & 0.116 & 0.031 & 0.031 \\
CO & 0.487 & 0.497 & 0.488 & 0.497 \\
SP & & -0.070 & -0.082 & -0.069 & -0.081 \\
Mean Ret & & 3.122 & 3.258 & 3.244 & 3.364 \\
StDev Ret & & 15.366 & 14.734 & 15.856 & 15.540 \\
\hline$c=0 \%$ & Sharpe & 0.203 & 0.221 & 0.205 & 0.217 \\
& $\Delta_{1}$ & & 23.1 & & 16.9 \\
& $\Delta_{10}$ & & $108.9^{*}$ & 0.200 & 0.211 \\
& Sharpe & 0.185 & 0.201 & & 16.8 \\
& $\Delta_{1}$ & & 22.0 & & $61.5^{*}$ \\
& $\Delta_{10}$ & & $107.8^{*}$ & 0.195 & 0.206 \\
& Sharpe & 0.166 & 0.181 & & 16.8 \\
& $\Delta_{1}$ & & 20.9 & & $61.5^{*}$ \\
\hline
\end{tabular}

Note: The table reports the long-horizon GMV portfolio results. The portfolios are re-balanced weekly or monthly based on the relevant weekly and monthly covariance matrix forecasts. Each panel shows turnover (TO), portfolio concentration ( $\mathrm{CO}$ ), and short positions (SP). The top panel also shows the average annualized return and standard deviation, while the bottom panel reports the standard deviation of the tracking error (TE). The table also reports the economic gains of switching from the standard HAR-DRD model to the HARQ-DRD model in annual basis points, $\Delta_{\gamma}$, for various transaction cost levels $c$ and risk aversion coefficients $\gamma$. Asterisks denote $\Delta_{\gamma}$ significantly different from zero at the $5 \%$ level.

matrix forecasts are shrunk toward some pre-determined target. We focus our comparisons on the HARQ-DRD model, which performed the best in the daily results discussed above.

\subsection{Weekly and monthly rebalancing}

The daily horizon underlying the results in the previous section might be expected to favor the new dynamic attenuation models, compared to the results obtained over longer weekly and monthly horizons, where the estimation errors in the realized covariances play a less important role. On the other hand, daily portfolio construction likely results in higher transaction costs than less frequent re-balancing. Correspondingly, some investors might be reluctant to change their positions on a daily basis, preferring instead a less frequent weekly or monthly re-balancing scheme. To investigate the robustness of our findings to the use of longer holding periods, we present the results in which we rely on the HAR(Q)-DRD models implemented at weekly and monthly frequencies.

To implement the models over these longer forecast horizons, we replace the one-day-ahead covariance matrix with the realized covariance matrix over the relevant horizon of interest. ${ }^{23}$ Specif-

\footnotetext{
${ }^{23}$ In the forecasting literature, this is commonly referred to as "direct" as opposed to "iterated" forecasting; see, e.g., Marcellino, Stock, and Watson (2006). In theory, if the daily model is correctly specified the iterated forecasts constructed from that model should be the most efficient. However, there is ample empirical evidence that even minor model mis-specifications tend to get amplified in iterated volatility forecasts, and as a result the direct forecast procedures often work better in practice; see, e.g., Andersen, Bollerslev, Diebold, and Labys (2003) and Sizova (2011).
} 
Table 9: Daily versus Weekly and Monthly Portfolio Allocations

\begin{tabular}{cccccc}
\hline & \multicolumn{2}{c}{ Weekly HARQ-DRD } & \multicolumn{2}{c}{ Monthly HARQ-DRD } \\
& & HAR-DRD & HARQ-DRD & HAR-DRD & HARQ-DRD \\
\hline $\mathrm{c}=0 \%$ & $\Delta_{1}$ & 31.2 & $113.7^{*}$ & $92.4^{*}$ & 174.9 \\
& $\Delta_{10}$ & -6.6 & $136.0^{*}$ & $152.7^{*}$ & $295.4^{*}$ \\
$\mathrm{c}=1 \%$ & $\Delta_{1}$ & -38.1 & $57.5^{*}$ & 1.7 & $97.3^{*}$ \\
& $\Delta_{10}$ & $-75.9^{*}$ & $79.7^{*}$ & $61.9^{*}$ & $217.7^{*}$ \\
$\mathrm{c}=2 \%$ & $\Delta_{1}$ & $-107.3^{*}$ & 1.3 & $-88.9^{*}$ & $61.1^{*}$ \\
& $\Delta_{10}$ & $-145.2^{*}$ & 23.5 & -28.8 & $140.0^{*}$ \\
\hline
\end{tabular}

Note: The table reports the economic gains of switching from weekly or monthly re-balanced GMV portfolios based on HARQ-DRD forecasts to a daily strategy based on HAR(Q)-DRD forecasts in annual basis points, $\Delta_{\gamma}$, for various transaction cost levels $c$ and risk aversion coefficients $\gamma$. Asterisks denote $\Delta_{\gamma}$ significantly different from zero at the $5 \%$ level.

ically, for the HAR formulation in equation (6),

$$
s_{t \mid t+h}=\theta_{0}+\theta_{1} s_{t-1}+\theta_{2} s_{t-5 \mid t-1}+\theta_{3} s_{t-22 \mid t-1}+\epsilon_{t}
$$

where $h=4$ and 21 for the weekly and monthly forecasts, respectively. We continue to rely on (overlapping) daily data in actually estimating the models. However, when forecasting the weekly (monthly) covariances, the weekly (monthly) lag tend to receive a relatively larger weight compared to its weight in the daily forecasting model. Correspondingly, we explicitly tailor the HARQ formulations to the specific forecast horizons, and only attenuate the relevant autoregressive parameters. ${ }^{24}$ That is, for the weekly HARQ model we keep $\theta_{1}$ and $\theta_{3}$ constant and replace $\theta_{2}$ with $\theta_{2, t}=\left(\theta_{2} \iota+\theta_{2 Q} \pi_{t-1 \mid t-5}\right)$, where $\pi_{t-1 \mid t-h} \equiv\left(\frac{1}{h} \sum_{i=1}^{h} \pi_{t-i}\right)^{1 / 2}$, while for the monthly model we keep $\theta_{1}$ and $\theta_{2}$ constant and replace $\theta_{3}$ with $\theta_{3, t}=\left(\theta_{3} \iota+\theta_{3 Q} \pi_{t-1 \mid t-22}\right)$. In parallel to the previous analysis, we assume that the investor relies on the resulting weekly $\Sigma_{t \mid t+4}$ and monthly $\Sigma_{t \mid t+21}$ covariance forecasts to construct GMV portfolios, re-balancing her positions at a weekly or monthly frequency, respectively.

The results for these longer horizon forecasting models and corresponding GMV portfolios averaged across all possible weekly (i.e., Monday-to-Monday, Tuesday-to-Tuesday, etc.) and monthly (i.e., 1st day-of-the-month, 2nd day-of-the-month, etc.) horizons are reported in Table 8. As expected, the estimated economic gains for the HARQ-DRD models relative to their conventional counterparts tend to be somewhat lower at the weekly level compared to the daily results reported in Table 5, and lower still at the monthly level. Moreover, turnover and portfolio concentrations are slightly higher for the "Q" models relative to their conventional counterparts. However, all of the $\Delta_{\gamma}$ s remain positive and statistically significant for all of the more risk averse cases, though not for the less risk averse cases. This again indicates the non-trivial benefits from using to the new dynamic attenuation models, even for longer holding periods.

To help illuminate the tradeoffs between the use of faster, but more costly-to-implement daily

\footnotetext{
${ }^{24}$ This also mirrors the simple univariate approach adopted in Bollerslev, Patton, and Quaedvlieg (2016).
} 
models, versus slower and cheaper-to-implement weekly and monthly models, Table 9 reports the estimated economic gains from shifting from the use of a daily HAR(Q)-DRD model to a weekly or monthly HARQ-DRD model. A positive $\Delta_{\gamma}$ in this table represents evidence in favor of daily modeling and re-balancing, while a negative $\Delta_{\gamma}$ supports the corresponding weekly or monthly model and less frequent re-balancing. Looking first at the results for the benchmark daily HAR-DRD portfolios, we see that in the absence of transaction costs, daily re-balancing generally improves on the weekly and monthly re-balanced HARQ-DRD portfolios, as reflected by the fact that all but one of the $\Delta_{\gamma}$ s for $c=0 \%$ are positive. However, the benefits of the daily HAR-DRD model over the weekly HARQ-DRD model are systematically wiped out when transaction costs are incorporated into the comparisons. This supports the common choice of coarser weekly or monthly re-balancing schemes as a simple way to help mitigate trading costs, when using simple forecasting models. In sharp contrast to this, all of the $\Delta_{\gamma}$ s for the our proposed daily HARQ-DRD model are positive, and all but two are statistically significant. This shows that by accounting for the time-varying measurement errors in the daily realized covariance estimates and obtaining more disciplined forecasts, the new dynamic attenuation models make daily re-balancing economically beneficial, even in the presence of transaction costs.

\subsection{Alternative ex-post shrinkage procedures}

The dynamic attenuation models endogenously shrink the influence of past realized covariances based on dynamically varying weights determined by an estimate of the reliability of the realized covariances. Alternative exogenous shrinkage type procedures, in which the forecasts themselves are shrunk to some target, have previously been proposed in the literature for mitigating the impact of estimation errors in the context of portfolio construction, notably the work by (Ledoit and Wolf, 2003, 2004a,b). This section compares the effectiveness of these shrinkage procedures to the new dynamic attenuation models in the construction of daily GMV portfolios, again focussing on the HARQ-DRD model.

Our implementation of the shrinkage procedures, is based on the traditional lower frequency estimators and theoretical set-up of Ledoit and Wolf (2003), as recently adapted to the highfrequency setting by Hautsch, Kyj, and Malec (2015). Specifically,

$$
H_{t \mid t-1}=\alpha_{t-1} F_{t-1}+\left(1-\alpha_{t-1}\right) S_{t-1}
$$

where $\alpha_{t-1}$ and $F_{t-1}$ denote the shrinkage intensity and shrinkage target, respectively. For $\alpha_{t-1} \equiv 0$ this obviously reduces to a random walk forecast based on the realized covariance matrix $S_{t-1}$, while for $\alpha_{t-1} \equiv 1$ the covariance matrix forecast is identically equal to the target matrix $F_{t-1}$. In the results reported on below, we rely on the estimate for the "optimal" shrinkage intensity formally derived by Ledoit and Wolf (2003) to determine the amount of shrinkage away from the random walk forecast. Several different choices have been proposed for the shrinkage target $F_{t}$. We consider three. 
Table 10: Shrinkage-based Portfolio Allocations

\begin{tabular}{|c|c|c|c|c|c|c|c|}
\hline & & \multirow{2}{*}{$\begin{array}{l}\text { HARQ- } \\
\text { DRD }\end{array}$} & \multirow[t]{2}{*}{ RW } & \multicolumn{3}{|c|}{ Shrunk RW } & \multirow[t]{2}{*}{$1 / \mathrm{N}$} \\
\hline & & & & Factor & Equicorr & Identity & \\
\hline TO & & 0.339 & 2.506 & 1.383 & 1.245 & 0.892 & 0.009 \\
\hline $\mathrm{CO}$ & & 0.497 & 0.867 & 0.620 & 0.596 & 0.453 & 0.316 \\
\hline $\mathrm{SP}$ & & -0.082 & -0.547 & -0.175 & -0.136 & -0.095 & 0.000 \\
\hline Mean Ret & & 4.371 & 3.649 & 4.497 & 4.600 & 1.437 & 1.044 \\
\hline StDev Ret & & 14.566 & 18.861 & 16.277 & 16.169 & 16.056 & 18.579 \\
\hline \multirow[t]{3}{*}{$c=0 \%$} & Sharpe & 0.300 & 0.193 & 0.276 & 0.284 & 0.090 & 0.056 \\
\hline & $\Delta_{1}$ & & $144.0^{*}$ & $13.8^{*}$ & 1.7 & $316.2^{*}$ & $399.2^{*}$ \\
\hline & $\Delta_{10}$ & & $790.1^{*}$ & $251.6^{*}$ & $223.8^{*}$ & $521.5^{*}$ & $997.1^{*}$ \\
\hline \multirow[t]{3}{*}{$c=1 \%$} & Sharpe & 0.241 & -0.141 & 0.062 & 0.090 & -0.050 & 0.055 \\
\hline & $\Delta_{1}$ & & $690.0^{*}$ & $276.8^{*}$ & $230.1^{*}$ & $455.5^{*}$ & $316.2^{*}$ \\
\hline & $\Delta_{10}$ & & $1334.5^{*}$ & $514.2^{*}$ & $451.9^{*}$ & $660.6^{*}$ & $914.1^{*}$ \\
\hline \multirow[t]{3}{*}{$c=2 \%$} & Sharpe & 0.183 & -0.476 & -0.152 & -0.104 & -0.190 & 0.054 \\
\hline & $\Delta_{1}$ & & $1236.1^{*}$ & $539.7^{*}$ & $458.6^{*}$ & $594.7^{*}$ & 233.1 \\
\hline & $\Delta_{10}$ & & $1879.1^{*}$ & $776.7^{*}$ & $680.0^{*}$ & $799.7^{*}$ & $831.1^{*}$ \\
\hline
\end{tabular}

Note: The table reports the results for different shrinkage procedures. The top panel shows turnover (TO), portfolio concentration ( $\mathrm{CO}$ ), short positions (SP), as well as the average annualized return and volatility. The bottom panel shows the economic gains of switching from the different alternatives to the HARQ-DRD-based forecasts in annual basis points, $\Delta_{\gamma}$, for various transaction cost levels $c$ and risk aversion coefficients $\gamma$. Asterisks denote $\Delta_{\gamma}$ significantly different from zero at the $5 \%$ level.

First, motivated by the single-factor model advocated in Ledoit and Wolf (2003), we consider a high-frequency-based realized equivalent. In particular, we equate the diagonal elements of $F_{t}$ to the realized variances for each of the stocks, while the off-diagonal elements are set equal to $S_{t}^{(m k t)} b_{t} b_{t}^{\prime}$, where $S_{t}^{(m k t)}$ and $b_{t}$ denote the realized variance of the market and the realized market betas for each of the stocks, respectively. This one-factor structure reduces the correlation among the stocks to their exposure to the common market factor. Second, following Voev (2008) among others, we rely on an equicorrelation structure based on the decomposition in equation (8), in which we restrict the correlations among all of the stocks to be the same. ${ }^{25}$ That is we set the target matrix to $D_{t} \bar{R}_{t} D_{t}$, where the off-diagonal elements in the $\bar{R}_{t}$ matrix is fixed at the average correlation among all of the stocks $\bar{\rho}_{t}=\frac{1}{N(N-1) / 2} \sum_{i=1}^{N-1} \sum_{j=i+1}^{N} \hat{\rho}_{i j, t}$. Finally, following Ledoit and Wolf (2004b), we consider the identity matrix as an all-purpose shrinkage target. For the target intensity $\alpha_{t-1} \equiv 1$ this reduces to an equal weighted portfolio of all the stocks. As shown by DeMiguel, Garlappi, and Uppal (2009b), this $1 / N$ portfolio is often difficult to beat, especially in large dimensions, and we include it as a final competitor.

The results from using these different shrinkage-based forecasts in the construction of daily GMV portfolios are reported in Table $10{ }^{26}$ As a reference, the first column reports the results based on the HARQ-DRD model, as previously reported in Table 5. The second column, labeled RW for random walk, reports the results corresponding to $\alpha_{t-1} \equiv 0$, followed by the results for the various

\footnotetext{
${ }^{25}$ This same idea also underlies the DECO model of Engle and Kelly (2012).

${ }^{26}$ The results for the minimum tracking error portfolios are again deferred to Appendix D.
} 
shrinkage targets and the equally-weighted $1 / N$ portfolio. Comparing the results for the different shrinkage portfolios, to the portfolios based on the random walk forecasts, reveals a significant reduction in both the turnover and the portfolio standard deviation. As such, this confirms the idea that the use of the historical realized covariance matrix leads to poor allocations, and that some regularization in the form of shrinkage generally improves on the portfolio performance. ${ }^{27}$

Abstracting from transaction costs, the Sharpe ratios for the factor- and equicorrelation-based shrinkage portfolios are also fairly close to the Sharpe ratio for the HARQ-DRD-based portfolios. However, the economic gains of shifting from any one of the shrinkage procedures to the HARQDRD model, as measured by the $\Delta_{\gamma} \mathrm{s}$, are always positive, and statistically significant in all but two cases. Moreover, including transaction cost into the comparisons results in massive economic gains of up to eight percent per year relative to the more traditional shrinkage-based portfolios. These results clearly underscore the advantages of dynamically incorporating the effect of measurement errors in the high-frequency-based realized covariances into the construction of covariance matrix forecasts and financial decisions, rather than simply shrink the covariance forecasts underlying the financial decisions to some naive target.

\section{Conclusion}

Fusing ideas from the econometrics literature on errors-in-variables with more recent results from the finance literature on the use of high-frequency intraday data and the estimation of realized risk measures, we provide a new and broadly applicable framework for more accurately forecasting financial risks. The basic idea behind the new approach is simple and intuitive: when current risks are measured with a high (low) degree of uncertainty, they should receive relatively low (high) weights in forecasts of future risks. Adapting various state-of-the-art realized covariance-based models to accommodate this feature and dynamically attenuate the influence of the past covariances not only results in on average more accurate, but importantly also more stable and from a practical perspective cheaper to implement, covariance forecasts. These improvements in turn translate into sizeable economic gains for a risk-averse investor seeking to minimize the variance of her equity portfolio or track the aggregate market.

The practical implementation of a host of other key concepts in risk management and asset pricing finance similarly depend on the ability to accurately forecast common risks as measured by the covariances of individual asset returns, or the covariances with appropriately defined benchmark portfolios. The new approach and simple-to-implement covariance forecasting models developed here thus holds the promise of empirically more accurate pricing models and improved financial decision making generally.

\footnotetext{
${ }^{27}$ The poor performance of the $1 / N$ portfolio reflects the fact that with "only" ten stocks, it is still possible to forecast the covariance matrix sufficiently precisely to beat a completely uninformative forecast.
} 


\section{References}

Aït-Sahalia, Y., Fan, J., Xiu, D., 2010. High-frequency covariance estimates with noisy and asynchronous financial data. Journal of the American Statistical Association 105 (492).

Andersen, T. G., Bollerslev, T., Christoffersen, P. F., Diebold, F. X., 2013. Financial risk measurement for financial risk management. In: Handbook of the Economics of Finance (eds. G. Constantinides, M. Harris and R. Stulz). Elsevier Inc., pp. 1127-1220.

Andersen, T. G., Bollerslev, T., Diebold, F. X., Labys, P., 2003. Modeling and forecasting realized volatility. Econometrica 71 (2), 579-625.

Andersen, T. G., Bollerslev, T., Diebold, F. X., Wu, G., 2006. Realized beta: Persistence and predictability. Advances in Econometrics 20, 1-39.

Andersen, T. G., Bollerslev, T., Huang, X., 2011. A reduced form framework for modeling volatility of speculative prices based realized variation measures. Journal of Econometrics 160 (2), 176-189.

Andersen, T. G., Bollerslev, T., Meddahi, N., 2004. Analytical evaluation of volatility forecasts. International Economic Review 45 (4), 1079-1110.

Andersen, T. G., Dobrev, D., Schaumburg, E., 2012. Jump-robust volatility estimation using nearest neighbor truncation. Journal of Econometrics 169 (1), 75-93.

Anderson, E. W., Cheng, A.-R. M., 2016. Robust Bayesian portfolio choice. Review of Financial Studies (forthcoming).

Baker, M., Bradley, B., Wurgler, J., 2011. Benchmarks as limits to arbitrage: Understanding the low-volatility anomaly. Financial Analysts Journal 67 (1), 40-54.

Bandi, F. M., Russell, J. R., Zhu, Y., 2008. Using high-frequency data in dynamic portfolio choice. Econometric Reviews 27 (1-3), 163-198.

Barndorff-Nielsen, O. E., Hansen, P. R., Lunde, A., Shephard, N., 2011. Multivariate realised kernels: consistent positive semi-definite estimators of the covariation of equity prices with noise and non-synchronous trading. Journal of Econometrics 162 (2), 149-169.

Barndorff-Nielsen, O. E., Shephard, N., 2004. Econometric analysis of realized covariation: High frequency based covariance, regression, and correlation in financial economics. Econometrica $72(3), 885-925$.

Bollerslev, T., 1990. Modelling the coherence in short-run nominal exchange rates: A multivariate Generalized ARCH model. Review of Economics and Statistics 72 (3), 498-505.

Bollerslev, T., Patton, A. J., Quaedvlieg, R., 2016. Exploiting the errors: A simple approach for improved volatility forecasting. Journal of Econometrics 192 (1), 1-18.

Brodie, J., Daubechies, I., De Mol, C., Giannone, D., Loris, I., 2009. Sparse and stable markowitz portfolios. Proceedings of the National Academy of Sciences 106 (30), 12267-12272.

Brown, D. B., Smith, J. E., 2011. Dynamic portfolio optimization with transaction costs: Heuristics and dual bounds. Management Science 57 (10), 1752-1770.

Chan, L. K., Karceski, J., Lakonishok, J., 1999. On portfolio optimization: Forecasting covariances and choosing the risk model. Review of Financial Studies 12 (5), 937-974. 
Chiriac, R., Voev, V., 2010. Modelling and forecasting multivariate realized volatility. Journal of Applied Econometrics 26 (6), 922-947.

Christensen, K., Kinnebrock, S., Podolskij, M., 2010. Pre-averaging estimators of the ex-post covariance matrix in noisy diffusion models with non-synchronous data. Journal of Econometrics 159 (1), 116-133.

Corsi, F., 2009. A simple approximate long-memory model of realized volatility. Journal of Financial Econometrics 7 (2), 174-196.

De Lira Salvatierra, I., Patton, A. J., 2015. Dynamic copula models and high frequency data. Journal of Empirical Finance 30, 120-135.

DeMiguel, V., Garlappi, L., Nogales, F. J., Uppal, R., 2009a. A generalized approach to portfolio optimization: Improving performance by constraining portfolio norms. Management Science 55 (5), 798-812.

DeMiguel, V., Garlappi, L., Uppal, R., 2009b. Optimal versus naive diversification: How inefficient is the 1/N portfolio strategy? Review of Financial Studies 22 (5), 1915-1953.

DeMiguel, V., Nogales, F. J., Uppal, R., 2014. Stock return serial dependence and out-of-sample portfolio performance. Review of Financial Studies 27 (4), 1031-1073.

Diebold, F. X., Mariano, R. S., 2002. Comparing predictive accuracy. Journal of Business \& Economic Statistics 20 (1).

Engle, R., Kelly, B., 2012. Dynamic equicorrelation. Journal of Business \& Economic Statistics $30(2), 212-228$.

Epps, T. W., 1979. Comovements in stock prices in the very short run. Journal of the American Statistical Association 74 (366a), 291-298.

Fan, J., Li, Y., Yu, K., 2012. Vast volatility matrix estimation using high-frequency data for portfolio selection. Journal of the American Statistical Association 107 (497), 412-428.

Fleming, J., Kirby, C., Ostdiek, B., 2001. The economic value of volatility timing. Journal of Finance 56 (1), 329-352.

Fleming, J., Kirby, C., Ostdiek, B., 2003. The economic value of volatility timing using realized volatility. Journal of Financial Economics 67 (3), 473-509.

Gonçalves, S., Meddahi, N., 2009. Bootstrapping realized volatility. Econometrica 77 (1), 283-306.

Han, Y., 2006. Asset allocation with a high dimensional latent factor stochastic volatility model. Review of Financial Studies 19 (1), 237-271.

Hansen, P. R., Lunde, A., 2005. A realized variance for the whole day based on intermittent highfrequency data. Journal of Financial Econometrics 3, 525-554.

Hansen, P. R., Lunde, A., 2006. Realized variance and market microstructure noise. Journal of Business \& Economic Statistics 24 (2), 127-161.

Hansen, P. R., Lunde, A., Nason, J. M., 2011. The model confidence set. Econometrica 79 (2), 453-497.

Hautsch, N., Kyj, L. M., Malec, P., 2015. Do high-frequency data improve high-dimensional port- 
folio allocations? Journal of Applied Econometrics 30 (2), 263-290.

Holtz-Eakin, D., Newey, W., Rosen, H. S., 1988. Estimating vector autoregressions with panel data. Econometrica 56 (6), 1371-1395.

Jagannathan, R., Ma, T., 2003. Risk reduction in large portfolios: Why imposing the wrong constraints helps. Journal of Finance 58 (4), 1651-1684.

Komunjer, I., Ng, S., 2014. Measurement errors in dynamic models. Econometric Theory 30 (1), 150-175.

Laurent, S., Rombouts, J. V., Violante, F., 2013. On loss functions and ranking forecasting performances of multivariate volatility models. Journal of Econometrics 173 (1), 1-10.

Lawrence, C. T., Tits, A. L., 2001. A computationally efficient feasible sequential quadratic programming algorithm. Siam Journal on Optimization 11 (4), 1092-1118.

Ledoit, O., Wolf, M., 2003. Improved estimation of the covariance matrix of stock returns with an application to portfolio selection. Journal of Empirical Finance 10 (5), 603-621.

Ledoit, O., Wolf, M., 2004a. Honey, I shrunk the sample covariance matrix. Journal of Portfolio Management 30 (4), 110-119.

Ledoit, O., Wolf, M., 2004b. A well-conditioned estimator for large-dimensional covariance matrices. Journal of Multivariate Analysis 88 (2), 365-411.

Li, J., 2015. Sparse and stable portfolio selection with parameter uncertainty. Journal of Business \& Economic Statistics 33 (3), 381-392.

Liu, Q., 2009. On portfolio optimization: How and when do we benefit from high-frequency data? Journal of Applied Econometrics 24 (4), 560-582.

Lunde, A., Shephard, N., Sheppard, K., 2015. Econometric analysis of vast covariance matrices using composite realized kernels and their application to portfolio choice. Journal of Business \& Economic Statistics (forthcoming).

Magnus, J. R., Neudecker, H., 1980. The elimination matrix: some lemmas and applications. SIAM Journal on Algebraic Discrete Methods 1 (4), 422-449.

Marcellino, M., Stock, J., Watson, M., 2006. A comparison of direct and iterated multistep ar methods for forecasting macroeconomic time series. Journal of Econometrics 135, 499-526.

Noureldin, D., Shephard, N., Sheppard, K., 2012. Multivariate high-frequency-based volatility (HEAVY) models. Journal of Applied Econometrics 27 (6), 907-933.

Oh, D. H., Patton, A. J., 2015. High dimension copula-based distributions with mixed frequency data. Journal of Econometrics, Forthcoming.

Pakel, C., Shephard, N., Sheppard, K., Engle, R. F., 2014. Fitting vast dimensional time-varying covariance models. Working Paper.

Patton, A. J., 2011. Volatility forecast comparison using imperfect volatility proxies. Journal of Econometrics 160 (1), 246-256.

Politis, D. N., Romano, J. P., 1994. The stationary bootstrap. Journal of the American Statistical Association 89 (428), 1303-1313. 
Pooter, M. d., Martens, M., Dijk, D. v., 2008. Predicting the daily covariance matrix for S\&P 100 stocks using intraday data-but which frequency to use? Econometric Reviews 27 (1-3), 199-229.

Sizova, N., 2011. Integrated variance forecasting: Model based vs. reduced form. Journal of Econometrics 162, 294-311.

Staudenmayer, J., Buonaccorsi, J. P., 2005. Measurement error in linear autoregressive models. Journal of the American Statistical Association 100 (471), 841-852.

Tu, J., Zhou, G., 2011. Markowitz meets Talmud: A combination of sophisticated and naive diversification strategies. Journal of Financial Economics 99, 204-215.

Varneskov, R., Voev, V., 2013. The role of realized ex-post covariance measures and dynamic model choice on the quality of covariance forecasts. Journal of Empirical Finance 20, 83-95.

Voev, V., 2008. Dynamic modelling of large-dimensional covariance matrices. In: High Frequency Financial Econometrics (eds. L. Bauwens, W. Pohlmeier and D. Veredas). Physica-Verlag, pp. 293-312.

White, H., 2000. A reality check for data snooping. Econometrica 68 (5), 1097-1126. 


\section{Appendix A. Multivariate Kernel theory and estimation}

Let the $N$-dimensional vector of discrete-time returns for the $i$ th intraday time-interval on day $t$ be denoted by $r_{i, t} \equiv P_{t-1+i \Delta}-P_{t-1+(i-1) \Delta}$. Further assume that we observe $M=1 / \Delta$ synchronized intra-daily returns for each of the $N$ assets. The Multivariate Kernel (MK) estimator of Barndorff-Nielsen, Hansen, Lunde, and Shephard (2011) (henceforth BHLS) is then defined as

$$
\mathrm{MK}_{t}=\sum_{h=-M}^{M} k(h / H) \Gamma_{h},
$$

where $\Gamma_{h}=\sum_{i=h+1}^{M} r_{i, t} r_{i-h, t}^{\prime}, \Gamma_{h}=\Gamma_{-h}^{\prime}$, and $k($.$) denotes an appropriate kernel function with$ bandwidth $H$. In the implementation here we use the Parzen kernel. The asymptotic theory of the MK further requires "jittering," as discussed in more detail in the BHLS paper. According to Theorem 2 of BHLS, it then follows that

$$
M^{1 / 5}\left(\operatorname{vech} \mathrm{MK}_{t}-\operatorname{vech} \Sigma_{t}\right) \rightarrow_{L s} M N\left(\omega_{t}, \Pi_{t}\right),
$$

where $\Pi_{t}=3.777 I Q_{t}$, and $I Q_{t}$ denotes the Integrated Quarticity. The bias term $\omega_{t}$ is negligibly, and we ignore it in our implementation. We estimate $\Pi_{t}$ based on pre-averaged data using the estimator of Barndorff-Nielsen and Shephard (2004). Specifically, defining $x_{i, t} \equiv \operatorname{vech}\left(\bar{r}_{i, t} \bar{r}_{i, t}^{\prime}\right)$, where $\bar{r}_{i, t}$ refers to the pre-averaged data,

$$
\widehat{I Q_{t}}=M \sum_{i=1}^{M-H} x_{i, t} x_{i, t}^{\prime}-\frac{M}{2} \sum_{j=1}^{M-H-1}\left(x_{i, t} x_{i+1, t}^{\prime}+x_{i+1, t}, x_{i, t}^{\prime}\right) .
$$

defines our consistent estimator for $I Q_{t}$.

\section{Appendix B. Simulation design}

In order to simulate empirically realistic sample paths of daily varying covariances, we draw from the empirical distribution of our data. To do so, we randomly select five of the ten stocks to obtain an MK-based covariance sample path of 2,000 consecutive days. For each day within that sample, we then simulate one-second returns with integrated covariance matrix equal to the MK estimate for that day. We do not allow for within day stochastic variation in the covariances, but we do include a diurnal pattern. The intraday volatility pattern is modeled by means of a diurnal U-shape function, $\sigma_{d}(u)$, by simulating form the following process using a standard Euler 
discretization scheme,

$$
\begin{aligned}
d P(u) & =S(u)^{1 / 2} d W(u), \\
S(u) & =\sigma_{d}(u) S \\
\sigma_{d}(u) & =C+A e^{-a u}+B e^{-b(1-u)},
\end{aligned}
$$

where, following Andersen, Dobrev, and Schaumburg (2012), we set the periodicity parameters to $A=0.75, B=0.25, C=0.88929198$, and $a=b=10$, respectively.

\section{Appendix C. Composite likelihood estimators}

The use of composite likelihoods in the estimation of large dimensional volatility models was recently popularized by Pakel, Shephard, Sheppard, and Engle (2014). To define the approach, let $V_{t}$ denote the $N \times N$ conditional covariance matrix for the $N \times 1$ vector of returns $r_{t}$. The standard quasi-likelihood obtained under the auxiliary assumption of conditional normality then takes the form,

$$
\log L(\psi ; r)=\sum_{t=1}^{T} \ell\left(\psi ; r_{t}\right)
$$

where,

$$
\ell\left(\psi ; r_{t}\right)=-\frac{1}{2} \log \left|V_{t}\right|-\frac{1}{2} r_{t}^{\prime} V_{t}^{-1} r_{t}
$$

If $N$ is of large dimension, this can be difficult and time-consuming to implement and also result in numerically unstable $V_{t}^{-1}$ matrices. The composite likelihood approach sidestep these problems by instead approximating the likelihood with a number of lower dimensional marginal densities, so that the dimension of the problem is reduced from $N$ to 2 . In the implementation adopted here, we rely on contiguous pairs, $Y_{1 t}=\left(r_{1, t}, r_{2, t}\right), Y_{2 t}=\left(r_{2, t}, r_{3, t}\right), \ldots, Y_{d-1, t}=\left(r_{d-1, t}, r_{d, t}\right)$, resulting in the composite likelihood,

$$
C L(\psi)=\frac{1}{T(N-1)} \sum_{j=1}^{N-1} \sum_{t=1}^{T} \ell_{j t}\left(\psi ; Y_{j t}\right) .
$$

Maximizing this much easier-to-implement $C L(\psi)$ function yields a consistent and asymptotically normal estimate for $\psi$. 


\section{Appendix D. Minimum tracking error portfolios}

Table D.1: No Short-Sale Minimum Tracking Error Portfolios

\begin{tabular}{|c|c|c|c|c|c|c|c|c|c|}
\hline & & HAR & HARQ & $\begin{array}{l}\text { HAR- } \\
\text { DRD }\end{array}$ & $\begin{array}{l}\text { HARQ- } \\
\text { DRD }\end{array}$ & EWMA & EWMAQ & HEAVY & HEAVYQ \\
\hline TO & & 0.172 & 0.102 & 0.134 & 0.114 & 0.063 & 0.045 & 0.080 & 0.056 \\
\hline $\mathrm{CO}$ & & 0.339 & 0.337 & 0.339 & 0.342 & 0.340 & 0.340 & 0.338 & 0.338 \\
\hline StDev TE & & 6.610 & 6.450 & 6.609 & 6.489 & 6.630 & 6.470 & 6.630 & 6.463 \\
\hline \multirow[t]{2}{*}{$c=0 \%$} & $\Delta_{1}$ & & 27.8 & & 38.0 & & 31.7 & & 39.0 \\
\hline & $\Delta_{10}$ & & 37.2 & & $45.1^{*}$ & & 41.1 & & 47.0 \\
\hline \multirow[t]{2}{*}{$c=1 \%$} & $\Delta_{1}$ & & $45.4^{*}$ & & $43.0^{*}$ & & 36.3 & & 36.8 \\
\hline & $\Delta_{10}$ & & $54.9^{*}$ & & $51.0^{*}$ & & 45.7 & & $46.7^{*}$ \\
\hline \multirow[t]{2}{*}{$c=2 \%$} & $\Delta_{1}$ & & $63.1^{*}$ & & $48.0^{*}$ & & $48.0^{*}$ & & $42.8^{*}$ \\
\hline & $\Delta_{10}$ & & $72.6^{*}$ & & $55.1^{*}$ & & $53.0^{*}$ & & $52.7^{*}$ \\
\hline
\end{tabular}

Note: The table reports the results for minimum tracking error portfolios that do not allow for short positions. The top panel shows the portfolio turnover (TO), portfolio concentration (CO), and annualized tracking error volatility. Standard deviations in bold indicate models that belong to the $90 \%$ model confidence set (MCS) of lowest ex-post daily volatility. The bottom panel reports the economic gains of switching from the conventional model to the Q-model in annual basis points, $\Delta_{\gamma}$, for various transaction cost levels $c$ and risk aversion coefficients $\gamma$. Asterisks denote $\Delta_{\gamma}$ significantly different from zero at the $5 \%$ level. 
Table D.2: Longer Horizon Tracking Portfolios

\begin{tabular}{|c|c|c|c|c|c|}
\hline & & \multicolumn{2}{|c|}{ Weekly } & \multicolumn{2}{|c|}{ Monthly } \\
\hline & & HAR-DRD & HARQ-DRD & HAR-DRD & HARQ-DRD \\
\hline TO & & 0.041 & 0.033 & 0.012 & 0.011 \\
\hline $\mathrm{CO}$ & & 0.339 & 0.342 & 0.339 & 0.342 \\
\hline SP & & 0.000 & 0.000 & 0.000 & 0.000 \\
\hline StDev TE & & 6.639 & 6.577 & 6.794 & 6.746 \\
\hline \multirow[t]{2}{*}{$c=0 \%$} & $\Delta_{1}$ & & $23.6^{*}$ & & 7.0 \\
\hline & $\Delta_{10}$ & & $27.3^{*}$ & & $9.9^{*}$ \\
\hline \multirow[t]{2}{*}{$c=1 \%$} & $\Delta_{1}$ & & $25.5^{*}$ & & 7.0 \\
\hline & $\Delta_{10}$ & & $29.2^{*}$ & & $9.9^{*}$ \\
\hline \multirow[t]{2}{*}{$c=2 \%$} & $\Delta_{1}$ & & $27.3^{*}$ & & 7.1 \\
\hline & $\Delta_{10}$ & & $31.0 *$ & & $10.0^{*}$ \\
\hline
\end{tabular}

Note: The table reports the long-horizon minimum tracking error portfolio results. The portfolios are re-balanced weekly or monthly based on the relevant weekly and monthly covariance matrix forecasts. The top panel shows turnover (TO), portfolio concentration (CO), short positions (SP), and annualized tracking error volatility. The bottom panel reports the economic gains of switching from the standard HAR-DRD model to the HARQ-DRD model in annual basis points, $\Delta_{\gamma}$, for various transaction cost levels $c$ and risk aversion coefficients $\gamma$. Asterisks denote $\Delta_{\gamma}$ significantly different from zero at the $5 \%$ level.

Table D.3: Weekly and Monthly versus Daily Tracking Portfolios

\begin{tabular}{lllclc}
\hline & \multicolumn{3}{c}{ Weekly } & \multicolumn{2}{c}{ Monthly } \\
& & HAR-DRD & HARQ-DRD & HAR-DRD & HARQ-DRD \\
\hline $\mathrm{c}=0 \%$ & $\Delta_{1}$ & 9.0 & $46.9^{*}$ & $46.1^{*}$ & $84.0^{*}$ \\
& $\Delta_{10}$ & 7.0 & $52.0^{*}$ & $55.1^{*}$ & $100.1^{*}$ \\
$\mathrm{c}=1 \%$ & $\Delta_{1}$ & -16.5 & $26.4^{*}$ & 15.2 & $58.1^{*}$ \\
& $\Delta_{10}$ & -18.5 & $31.5^{*}$ & 24.2 & $74.2^{*}$ \\
$\mathrm{c}=2 \%$ & $\Delta_{1}$ & $-41.9^{*}$ & 5.9 & -15.7 & $37.7^{*}$ \\
& $\Delta_{10}$ & $-43.9^{*}$ & $11.1^{*}$ & -6.7 & $48.3^{*}$ \\
\hline
\end{tabular}

Note: The table reports the economic gains of switching from weekly or monthly re-balanced minimum tracking error portfolios based on HARQ-DRD model forecasts to a daily strategy based on $\operatorname{HAR}(\mathrm{Q})-\mathrm{DRD}$ forecasts in annual basis points, $\Delta_{\gamma}$, for various transaction cost levels $c$ and risk aversion coefficients $\gamma$. Asterisks denote $\Delta_{\gamma}$ significantly different from zero at the $5 \%$ level. 
Table D.4: Shrinkage-based Tracking Portfolios

\begin{tabular}{|c|c|c|c|c|c|c|c|}
\hline & & \multirow[t]{2}{*}{ HARQ-DRD } & \multirow[t]{2}{*}{ RW } & \multicolumn{3}{|c|}{ Shrunk RW } & \multirow[t]{2}{*}{$1 / \mathrm{N}$} \\
\hline & & & & $\begin{array}{l}\text { Factor } \\
\text { Model }\end{array}$ & Equicol & n Identity & \\
\hline TO & & 0.114 & 1.128 & 0.618 & 0.555 & 0.415 & 0.009 \\
\hline $\mathrm{CO}$ & & 0.342 & 0.461 & 0.380 & 0.375 & 0.344 & 0.316 \\
\hline $\mathrm{SP}$ & & 0.000 & -0.087 & -0.004 & -0.001 & -0.004 & 0.000 \\
\hline StDev TE & & 6.521 & 8.577 & 7.243 & 7.085 & 7.060 & 7.146 \\
\hline \multirow[t]{2}{*}{$c=0 \%$} & $\Delta_{1}$ & & $254.5^{*}$ & $41.9^{*}$ & $-60.4^{*}$ & $152.4^{*}$ & $36.9^{*}$ \\
\hline & $\Delta_{10}$ & & $394.3^{*}$ & $86.7^{*}$ & -25.8 & $185.5^{*}$ & $85.4^{*}$ \\
\hline \multirow[t]{2}{*}{$c=1 \%$} & $\Delta_{1}$ & & $510.1^{*}$ & $168.9^{*}$ & $50.7^{*}$ & $228.2^{*}$ & $20.5^{*}$ \\
\hline & $\Delta_{10}$ & & $649.8^{*}$ & $213.6^{*}$ & $85.2^{*}$ & $261.3^{*}$ & $59.1^{*}$ \\
\hline \multirow[t]{2}{*}{$c=2 \%$} & $\Delta_{1}$ & & $765.7^{*}$ & $295.9^{*}$ & $161.8^{*}$ & $304.0^{*}$ & -5.8 \\
\hline & $\Delta_{10}$ & & $905.3^{*}$ & $340.6^{*}$ & $196.3^{*}$ & $337.1^{*}$ & $32.7^{*}$ \\
\hline
\end{tabular}

Note: The table reports minimum tracking error portfolios formed based on exogenously shrunk forecasts. The top panel shows turnover (TO), portfolio concentration $(\mathrm{CO})$, short position( $\mathrm{SP}$ ), as well as the average annualized tracking error volatility. The bottom panel shows the economic gains of switching from the alternatives to the HARQ-DRD model forecasts in annual basis points, $\Delta_{\gamma}$, for various transaction cost levels $c$ and risk aversion coefficients $\gamma$. Asterisks denote $\Delta_{\gamma}$ significantly different from zero at the $5 \%$ level. 
2015-53: Mark Podolskij and Nopporn Thamrongrat: A weak limit theorem for numerical approximation of Brownian semi-stationary processes

2015-54: $\quad$ Peter Christoffersen, Mathieu Fournier, Kris Jacobs and Mehdi Karoui: Option-Based Estimation of the Price of Co-Skewness and Co-Kurtosis Risk

2015-55 Kadir G. Babaglou, Peter Christoffersen, Steven L. Heston and Kris Jacobs: Option Valuation with Volatility Components, Fat Tails, and Nonlinear Pricing Kernels

2015-56: $\quad$ Andreas Basse-O'Connor, Raphaël Lachièze-Rey and Mark Podolskij: Limit theorems for stationary increments Lévy driven moving averages

2015-57: $\quad$ Andreas Basse-O'Connor and Mark Podolskij: On critical cases in limit theory for stationary increments Lévy driven moving averages

2015-58: $\quad$ Yunus Emre Ergemen, Niels Haldrup and Carlos Vladimir Rodríguez-Caballero: Common long-range dependence in a panel of hourly Nord Pool electricity prices and loads

2015-59: $\quad$ Niels Haldrup and J. Eduardo Vera-Valdés: Long Memory, Fractional Integration, and Cross-Sectional Aggregation

2015-60: Mark Podolskij, Bezirgen Veliyev and Nakahiro Yoshida: Edgeworth expansion for the pre-averaging estimator

2016-01: Matei Demetrescum, Christoph Hanck and Robinson Kruse: Fixed-b Inference in the Presence of Time-Varying Volatility

2016-02: $\quad$ Yunus Emre Ergemen: System Estimation of Panel Data Models under LongRange Dependence

2016-03: Bent Jesper Christensen and Rasmus T. Varneskov: Dynamic Global Currency Hedging

2016-04: Markku Lanne and Jani Luoto: Data-Driven Inference on Sign Restrictions in Bayesian Structural Vector Autoregression

2016-05: $\quad$ Yunus Emre Ergemen: Generalized Efficient Inference on Factor Models with Long-Range Dependence

2016-06: $\quad$ Girum D. Abate and Luc Anselin: House price fluctuations and the business cycle dynamics

2016-07: $\quad$ Gustavo Fruet Dias, Cristina M. Scherrer and Fotis Papailias: Volatility Discovery

2016-08 N. Haldrup, O. Knapik and T. Proietti: A generalized exponential time series regression model for electricity prices

2016-09: Ole E. Barndorff-Nielsen: Assessing Gamma kernels and BSS/LSS processes

2016-10: $\quad$ Tim Bollerslev, Andrew J. Patton and Rogier Quaedvlieg: Modeling and Forecasting (Un)Reliable Realized Covariances for More Reliable Financial Decisions 